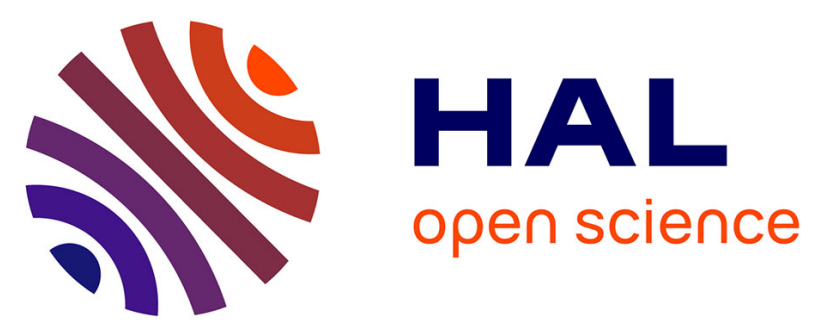

\title{
Elastic fields due to dislocations in anisotropic bi-and tri-materials: applications to discrete dislocation pile-ups at grain boundaries
}

Xiaolei Chen, Thiebaud Richeton, Christian Motz, Stéphane Berbenni

\section{To cite this version:}

Xiaolei Chen, Thiebaud Richeton, Christian Motz, Stéphane Berbenni. Elastic fields due to dislocations in anisotropic bi-and tri-materials: applications to discrete dislocation pile-ups at grain boundaries. International Journal of Solids and Structures, 2019, 164, pp.141-156. 10.1016/j.ijsolstr.2019.01.020 . hal-02360489

\section{HAL Id: hal-02360489 \\ https://hal.science/hal-02360489}

Submitted on 12 Nov 2019

HAL is a multi-disciplinary open access archive for the deposit and dissemination of scientific research documents, whether they are published or not. The documents may come from teaching and research institutions in France or abroad, or from public or private research centers.
L'archive ouverte pluridisciplinaire HAL, est destinée au dépôt et à la diffusion de documents scientifiques de niveau recherche, publiés ou non, émanant des établissements d'enseignement et de recherche français ou étrangers, des laboratoires publics ou privés. 


\title{
Elastic fields due to dislocations in anisotropic bi- and tri-materials: applications to discrete dislocation pile-ups at grain boundaries
}

\author{
Xiaolei Chen ${ }^{1,2}$, Thiebaud Richeton ${ }^{1 *}$, Christian Motz ${ }^{2}$, Stéphane Berbenni ${ }^{1}$ \\ ${ }^{1}$ Université de Lorraine, CNRS, Arts et Métiers ParisTech, LEM3, F-57070 Metz, France \\ ${ }^{2}$ Department of Materials Science and Methods, Saarland University, Germany
}

\begin{abstract}
Elastic fields due to single dislocations and dislocation pile-ups are computed in heterogeneous media like bi-materials, half-spaces and tri-materials thanks to the Leknitskii-Eshelby-Stroh formalism for two-dimensional anisotropic elasticity. The tri-material configuration allows to consider grain boundary regions with finite thickness and specific stiffness. The effects of these parameters are first studied in the case of a single dislocation in a Ni bicrystal. Image forces may arise because of both dissimilar grain orientations and the presence of a finite grain boundary region. In particular, it is shown that the Peach-Koehler force projected along the dislocation glide direction can exhibit a change of sign with the dislocation position. Therefore, an equilibrium position in the absence of applied stress can be found by coupling an attractive compliant grain boundary region with a repulsive orientation of the adjacent crystal, or a repulsive stiff grain boundary region with an attractive orientation. Regarding dislocation pile-ups, it is shown that the resolved shear stress scales approximately with the inverse of the square root distance from the leading dislocation in the pile-up. This scaling law remains valid in anisotropic elasticity for the chosen heterogeneous media. Both the grain boundary stiffness and grains misorientation influence pile-up length and resolved shear stress, but the effect of misorientation is clearly seen to be predominant. In the case where the leading dislocation is unlocked, the resolved shear stress at a given position in the neighboring grain is reduced when the grain boundary stiffness is increased due to the pushing back of dislocations from the grain boundary.
\end{abstract}




\section{Keywords}

Bi-crystal; Grain boundary; Anisotropic elasticity; Dislocation pile-up; Edge dislocation; Image force; Resolved shear stress

\section{Introduction}

The mechanical properties of polycrystals are greatly dependent on the interaction mechanisms between dislocations and grain boundaries (dislocation transmission or absorption at grain boundary, formation of a dislocation pile-up, etc). Beyond the specific details of these mechanisms, dislocations always interact with grain boundaries through their elastic fields. In heterogeneous media like bi-crystals containing grains with different orientations, image stresses are indeed appended to the self dislocation stress field (i.e. the one for an infinite homogeneous medium) in order to satisfy the boundary conditions at interfaces. The image forces can either attract the dislocation towards the grain boundary or conversely push the dislocation away. In case of a dislocation pile-up, image forces can especially influence the positions of the dislocations and the stress field caused in the adjacent crystal. Such forces can be considered as configurational forces (Asaro and Lubarda, 2006; Maugin, 2011). Moreover, real grain boundaries have finite width and their specific elastic properties may then constitute an additional source of image forces.

Elastic fields due to the presence of singularities like dislocation and line force in anisotropic infinite homogeneous elastic medium were originally developed by Eshelby et al. (1953), Stroh (1958) and Leknitskii (1963). The so-called Leknitskii-Eshelby-Stroh (LES) analytical formalism (or sextic equation formalism) involves solving a six-dimensional equation and considers complex variable techniques. It is very efficient but cannot handle the case of a completely isotropic crystal since the problem becomes singular due to repeated eigenvalues (see e.g. Ting, 1996). To circumvent this issue, an integral formalism was also developed, 
which is based on Green's function technique and does not require to find eigenvalues and eigenvectors (Barnett and Lothe, 1973; Bacon et al., 1979).

The general problem of anisotropic bi-materials and half-spaces with singularities was firstly solved by Gemperlova, Tucker and co-workers (Tucker and Crocker, 1968; Gemperlová and Saxl, 1968; Gemperlová, 1968, Tucker, 1969), then by Tewary et al. (1989) followed by Suo (1990) who used analytic continuation arguments. Ting (1992), Ting and Barnett (1993), Chu and Pan (2014) used Green's function technique for anisotropic media (Ting, 1996). Considering perfectly bonded interfaces, all these methods provide identical solutions and results. It is also noteworthy that many other authors considered the particular case of an interfacial dislocation in a bi-material with many applications to micromechanics problems including interfacial elasticity and interfacial cracks (e.g., Nakahara and Willis, 1973; Barnett and Lothe, 1974; Chou et al., 1975; Hirth et al., 1979; Bonnet and Dupeux, 1980; Qu and Bassani, 1989; Qu and Li, 1991; Wu et al., 2003; Sigaeva and Schiavone, 2014; Juan and Dingreville, 2017a,b). Besides, it can be underlined that the case of imperfect interfaces was already addressed by Tucker (1969) who obtained elastic field solutions for both perfectly bonded and freely slipping interfaces. Most recently, elastic Green's functions were derived in anisotropic bi-materials by Pan (2003) in three dimensions while considering different kinds of imperfect interface models and by Juan and Dingreville, (2017b) while accounting for interfacial elasticity. Furthermore, it must be noticed also that elastic field solutions due to three-dimensional dislocation loops in anisotropic bi-materials exist (e.g., Han and Ghoniem (2005), Chu et al. (2012)).

The elastic fields of dislocations in anisotropic layered media were studied by using the Stroh formalism in conjunction with the Fourier integral transform, e.g. Alshits and Kirchner (1995), Han and Ghoniem (2005), or in conjunction with the image decomposition method using singular Cauchy integral equations, e.g. Wang et al. (2007). An alternative interesting method is to implement the analytical solutions based on recurrence formulas as derived by Choi and Earmme in anisotropic (Choi and Earmme, 2002a) and isotropic (Choi and Earmme, 2002b) tri-materials. To perform their derivations, Choi and Earmme (2002a) considered an alternating 
technique to satisfy boundary conditions at both interfaces in combination with the analytic continuation method (Suo, 1990; Hwu, 2010). In particular, Choi and Earmme (2002a) applied their recurrence formulas to find the elastic fields due to the presence of a dislocation in a finite epitaxial layer lying on an infinite substrate, the third (top) material being a free space.

Dislocation pile-up at grain boundaries is an important inelastic deformation mechanism in polycrystalline materials that has been observed in metals using Transmission Electron Microscopy (TEM) (e.g., Lee et al., 1990) or using stage-I fatigue crack experiment (e.g., Schäfer et al., 2016). The grain size dependence of the flow stress of polycrystals, which is well known as the Hall-Petch relationship, can be explained by the stresses caused by dislocation pile-ups at grain boundaries (Eshelby et al., 1951; Li and Liu, 1967). Stress concentration built up at the head of dislocation pile-ups may be the driving force for slip transfer and activation of new dislocation sources in the neighboring grain. In order to compute the elastic fields of dislocation pile-ups at grain boundaries, two-dimensional elasticity is generally considered and dislocations are represented as a set of identical parallel straight infinite dislocations lying in the same slip plane. Both discrete and continuous-based theories of pile-ups have been developed (e.g., Chou and Li, 1969; Voskoboinikov et al., 2007). The calculation of equilibrium positions of discrete dislocations in a pile-up was firstly performed by Eshelby et al. (1951) in an isotropic homogeneous elastic crystal. The equilibrium position solutions were described as the roots of a generalized Laguerre polynomial. On the other hand, Leibfried (1951) studied continuous pile-ups where discrete dislocations were replaced by a continuously distributed dislocation density. From Eshelby's work, Mitchell et al. (1965) computed dislocation equilibrium positions for discrete pile-up in anisotropic homogeneous crystals.

Regarding dislocation pile-ups in heterogeneous media, many works dealt with planar or circular bimetallic interfaces but mostly in isotropic elasticity (Chou, 1966; Barnett, 1967; Kuang and Mura, 1968; Kuan and Hirth, 1976; Öveçoğlu et al., 1987; Lubarda, 2017). In particular, Lubarda (2017) recently studied the effect of the number dislocations, the applied shear stress, the size of the inhomogeneity and the degree of mechanical contrast on the equilibrium positions of edge dislocation pile-up against a circular inhomogeneity or a 
bimetallic interface. He also evaluated the configurational force on a circular inhomogeneity and the stress concentration caused by the pile-up against different interfaces, which is of importance for the study of interface cracking. A few works about pile-ups are however concerned with heterogeneous anisotropic elasticity, as the ones of Vagera (1971) for continuous pile-ups and Wagoner for discrete pile-ups (Wagoner, 1981). Wagoner (1981) found dislocation positions of an equilibrated pile-up in an anisotropic bicrystal using a numerical iterative relaxation scheme with a first locked dislocation at small distance from grain boundary. The iterative relaxation scheme minimizes the Peach-Koehler force acting on each dislocation along the slip direction. Up to now, a few discrete dislocation dynamics studies incorporate elastic anisotropy framework to integrate image forces in bi- and tri-materials (see e.g. Shishvan et al., 2011).

In the present paper which focuses only on perfectly bonded interfaces, a numerical method similar to Wagoner's one is used to calculate equilibrium positions and stress fields of dislocation pile-ups in anisotropic heterogeneous medium like bi-materials, half-spaces and tri-materials. In the latter case, the recurrence formulas of Choi and Earmme (2002a) are used while the finite medium stands for the grain boundary region. The paper is organized as follows. Notation conventions are settled in section 2 and the LES formalism for two-dimensional anisotropic elasticity is recalled in section 3. Section 4 deals with the issue of a single straight dislocation in anisotropic homogeneous medium and anisotropic heterogeneous medium like bi-materials and half-spaces (Suo, 1990; Hwu, 2010). Section 5 recalls the alternating technique method of Choi and Earmme (2002a) to find the solutions for a single straight dislocation in an anisotropic tri-material. Moreover, two new criteria for the convergence of the solutions are proposed. Section 6 is dedicated to the discrete dislocation pile-up theory. In particular, the theory of Eshelby et al. (1951) on the stress concentration caused by a pile-up is briefly recalled. Then, Wagoner's method is used to study discrete pile-ups in anisotropic homogeneous media, bi-materials, half-spaces and it is extended to tri-materials. In section 7, results and discussions are reported. First of all, computation configurations are explained, then a new convergence study for tri-material configurations is proposed. The effects of grain boundary modelling characteristics as well as image forces are 
studied for single dislocation configurations. Secondly, the results about dislocation pile-ups in anisotropic bi-crystals, using bi- and tri-material models, are reported and discussed when the leading dislocation is arbitrarily locked and when it is unlocked and equilibrated thanks to a repulsive image force. Finally, concluding remarks are provided in section 8 .

\section{Notations}

A bold lowercase letter like $\boldsymbol{v}$ denotes a vector and a bold uppercase letter like $\boldsymbol{W}$ denotes a $3 \times 3$ matrix. So $\boldsymbol{W} \boldsymbol{v}$ denotes the action of the matrix $\boldsymbol{W}$ on the vector $\boldsymbol{v}$, producing a vector. The symbol $\times$ represents the cross product. The superscript $\mathrm{T}$ denotes the transpose of a matrix. The Einstein convention over repeated indices is used when indices are underlined, e.g.: $A_{i \underline{j}} f_{\underline{j}}\left(z_{\underline{j}}\right)=A_{i 1} f_{1}\left(z_{1}\right)+A_{i 2} f_{2}\left(z_{2}\right)+A_{i 3} f_{3}\left(z_{3}\right)$ but $f_{j}\left(z_{j}\right) \neq f_{1}\left(z_{1}\right)+$ $f_{2}\left(z_{2}\right)+f_{3}\left(z_{3}\right)$ and $\boldsymbol{f}=\left[f_{1}(z), f_{2}(z), f_{3}(z)\right]^{\mathrm{T}}$ is a vector function, while the $f_{j}$ are arbitrary functions of their arguments. Besides, the notation $\bar{f}(z)=\overline{f(\bar{z})}$ is used for the conjugate of a complex function.

$\boldsymbol{\sigma}$ is the second-order Cauchy stress tensor with $\sigma_{i j}=\sigma_{j i}, \boldsymbol{u}$ is the displacement vector, and $\boldsymbol{\varepsilon}$ is the second-order strain tensor defined by $\varepsilon_{i j}=\frac{1}{2}\left(u_{i, j}+u_{j, i}\right)$, where the spatial derivative with respect to a Cartesian coordinate is indicated by a comma followed by the component index. $\boldsymbol{C}$ denotes the elastic stiffness tensor, which is a fourth-order tensor with classic symmetries: $C_{\mathrm{ijkl}}=C_{\mathrm{jikl}}=C_{\mathrm{ijlk}}=C_{\mathrm{klij}}$.

\section{Leknitskii-Eshelby-Stroh (LES) formalism for two-dimensional anisotropic elasticity}

Details about the LES formalism can be found in many textbooks, e.g., Ting (1996) or Hwu (2010). Below, the main equations are given in order to introduce clearly the variables used later. A Cartesian reference frame $\left(\boldsymbol{e}_{1}, \boldsymbol{e}_{2}, \boldsymbol{e}_{3}\right)$ such that $\boldsymbol{e}_{3}=\boldsymbol{e}_{1} \times \boldsymbol{e}_{2}$ is considered throughout the paper. Assuming that $u_{k}$ depends on $\left(x_{1}, x_{2}\right)$ only, the balance of linear momentum in the absence of body forces coupled with the Hooke's law in linear anisotropic 
elasticity $\left(C_{i \underline{j k l}} u_{\underline{k}, \underline{l j}}=0\right)$ leads to:

$$
C_{i 1 \underline{k} 1} u_{\underline{k}, 11}+\left(C_{i 1 \underline{k} 2}+C_{i 2 \underline{k} 1}\right) u_{\underline{k}, 12}+C_{i 2 \underline{k} 2} u_{\underline{k}, 22}=0
$$

Without loss of generality, it has been shown that the solution of Eq. ( 1 ) can be expressed as (Eshelby et al., 1953; Stroh, 1958):

$$
u_{k}=a_{k} f(z) \text { with } z=x_{1}+p x_{2}
$$

where $f$ is an arbitrary scalar function of $z$ and $a_{k}$ and $p$ are constants to be determined. Then, by substitution, a sextic equation for $p$ is obtained:

$$
\left(C_{i 1 \underline{k} 1}+p\left(C_{i 1 \underline{k} 2}+C_{i 2 \underline{k} 1}\right)+p^{2} C_{i 2 \underline{k} 2}\right) a_{\underline{k}}=0
$$

since a nontrivial solution of $a_{k}$ exists only if the determinant of Eq. ( 3 ) is zero. The six roots (defined hereafter as the material eigenvalues) actually consist of three pairs of complex conjugate roots due to the positive definiteness of the strain energy (Eshelby et al., 1953). If $p_{\alpha}$ and $a_{k \alpha}(\alpha=1 \ldots 6)$ denote the eigenvalues and the associated eigenvector components, it is hereafter considered that:

$$
\operatorname{Im}\left(p_{\alpha}\right)>0, p_{\alpha}=\overline{p_{\alpha+3}}, a_{k \alpha}=\overline{a_{k(\alpha+3)}} \text { with } \alpha=1,2,3
$$

Assuming generally that the roots $p_{\alpha}$ are distinct, the displacement vector is then obtained by the superposition of the six solutions:

$$
u_{i}=2 \operatorname{Re}\left[A_{i \underline{j}} f_{\underline{j}}\left(z_{\underline{j}}\right)\right]
$$

where $\boldsymbol{A}$ is a $3 \times 3$ matrix containing the eigenvector components $a_{k \alpha}(\alpha=1,2,3)$ and $z_{j}=x_{1}+p_{j} x_{2}$. Finally, the stresses can be derived from a stress function vector $\phi_{i}$ obtained as:

$$
\phi_{i}=2 \operatorname{Re}\left[B_{i \underline{j}} f_{\underline{j}}\left(z_{\underline{j}}\right)\right]
$$

where $\boldsymbol{B}$ is a $3 \times 3$ matrix defined as:

$$
B_{i j}=\left(C_{i 2 \underline{k} 1}+p_{j} C_{i 2 \underline{k} 2}\right) A_{\underline{k} j}
$$

such that the $\boldsymbol{e}_{3}$-independent stresses are: 


$$
\sigma_{i 1}=-\partial \phi_{i} / \partial x_{2}, \sigma_{i 2}=\partial \phi_{i} / \partial x_{1}
$$

If needed, component $\sigma_{33}$ can be computed from the generalized Hooke's law and under plane strain assumption. It is noteworthy that $p_{\alpha}$, as well as the matrices $\boldsymbol{A}$ and $\boldsymbol{B}$, are complex quantities, which depend only on the anisotropic elastic stiffness tensor $\boldsymbol{C}$. Matrices $\boldsymbol{A}$ and $\boldsymbol{B}$ are non-singular when the six complex roots $p_{\alpha}$ are all distinct or for specific non-degenerate cases (Ting and Chyanbin, 1988). Properties of $\boldsymbol{A}$ and $\boldsymbol{B}$ (normalization, orthogonality) were discussed by Stroh $(1958,1962)$, Barnett and Lothe (1974) and Chadwick and Smith (1977).

\section{Single straight dislocation in anisotropic elasticity}

In the following, an infinite straight dislocation with Burgers vector $\boldsymbol{b}$ and whose line $\boldsymbol{t}$ is parallel to the $\boldsymbol{e}_{3}$ direction (i.e. the $x_{3}$ axis in Figure 1) is considered at the position $\left(X_{1}, X_{2}\right)$. Through the paper, the so-called FS/RH (Finish Start/Right Hand) convention (Hirth and Lothe, 1982) is used to define the direction of the dislocation line (see Figure 1).

\subsection{Homogeneous medium}

For a homogeneous anisotropic medium, the function vector $\boldsymbol{f}$ is denoted $\boldsymbol{f}^{\mathbf{0}}$. It is derived by considering the boundary condition associated to the presence of the dislocation with Burgers vector $\boldsymbol{b}$ in the absence of remotely applied force (Eshelby et al., 1953; Stroh, 1958):

$$
\int_{\mathcal{C}} \boldsymbol{d u}=\boldsymbol{b}
$$

where $\mathcal{C}$ is any closed curve (Burgers circuit) enclosing the position $\left(X_{1}, X_{2}\right)$. Following Suo (1990), the general form of $f_{j}^{0}\left(z_{i}\right)$ is given by:

$$
f_{j}^{0}\left(z_{i}\right)=q_{j}^{0} \ln \left(z_{i}-s_{j}\right)
$$

where $z_{i}=x_{1}+p_{i} x_{2}, s_{j}=X_{1}+p_{j} X_{2}$ and $\boldsymbol{q}^{\mathbf{0}}$ is a complex vector expressed as: 


$$
\boldsymbol{q}^{\mathbf{0}}=-\frac{1}{2 \pi} i\left(\boldsymbol{B}^{\mathrm{T}} \boldsymbol{b}\right)
$$

\subsection{Heterogeneous medium: bi-material}

Let us now consider an anisotropic bi-material (or bi-crystal) with a perfectly bonded interface whose normal is along the $\boldsymbol{e}_{2}$ direction (i.e. the $x_{2}$ axis in Figure 1). The dislocation line is supposed to be located in the upper material I $\left(x_{2}>0\right)$ (Figure 1). In addition to Eq. ( 9 ), the function vector $\boldsymbol{f}$ should also satisfy the continuity conditions at the perfect interface, i.e. the continuity of displacements and forces across the interface (Suo, 1990).

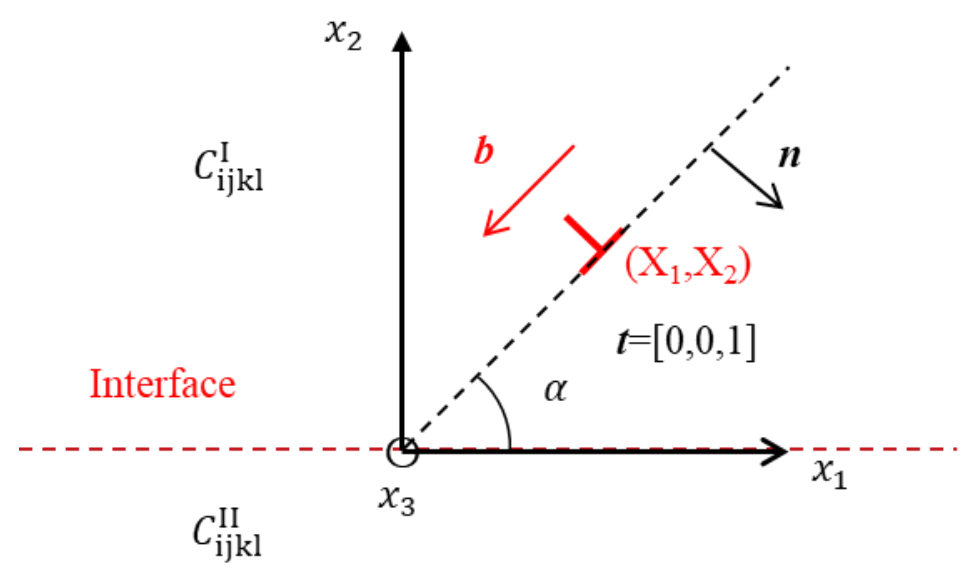

Figure 1. A single straight edge dislocation in a bi-material.

Following the Suo's method (Suo, 1990), the function vector $f_{j}\left(z_{j}\right)$ to be used in Eqs. ( 5 ) and ( 6 ) is expressed as:

$$
f_{j}\left(z_{j}\right)= \begin{cases}f_{j}^{0}\left(z_{j}\right)+f_{j}^{\mathrm{I}}\left(z_{j}\right) & \text { if } z_{j} \in \mathrm{I}\left(x_{2}>0\right) \\ f_{j}^{\mathrm{II}}\left(z_{j}\right) & \text { if } z_{j} \in \mathrm{II}\left(x_{2}<0\right)\end{cases}
$$

where $f_{j}^{0}\left(z_{j}\right)$ is the function of the homogeneous problem calculated from Eq. ( 10 ) considering the elastic stiffness tensor $\boldsymbol{C}^{\mathbf{I}}$ of the upper material I and $s_{j}=s_{j}^{\mathrm{I}}=X_{1}+p_{j}^{\mathrm{I}} X_{2}$. 
Moreover, it must be noticed that if $z_{j} \in \mathrm{I}, z_{j}=z_{j}^{\mathrm{I}}=x_{1}+p_{j}^{\mathrm{I}} x_{2}$ and if $z_{j} \in \mathrm{II}, z_{j}=z_{j}^{\mathrm{II}}=$ $x_{1}+p_{j}^{\mathrm{II}} x_{2}$ where $p_{j}^{\mathrm{I}}$ and $p_{j}^{\mathrm{II}}$ are components of the vectors containing the roots with positive imaginary parts of the sextic equation (Eq. ( 3 )) solved in crystals I and II, respectively. The functions $f_{j}^{\mathrm{I}}\left(z_{j}\right)$ and $f_{j}^{\mathrm{II}}\left(z_{j}\right)$ are determined from continuity conditions at the interface and analytical continuation arguments. The reader is referred to (Suo, 1990) and (Hwu, 2010) to find details about these derivations. As a result, the following expressions of $f_{i}^{\mathrm{I}}\left(z_{i}\right)$ and $f_{i}^{\mathrm{II}}\left(z_{i}\right)$ are obtained:

$$
\left\{\begin{array}{l}
f_{i}^{\mathrm{I}}\left(z_{i}\right)=\overline{V_{i j}^{\mathrm{I}, \mathrm{II}}} \overline{f_{j}^{0}}\left(z_{i}\right) \text { if } z_{i} \in \mathrm{I} \\
f_{i}^{\mathrm{II}}\left(z_{i}\right)=W_{i \underline{j}}^{\mathrm{I}, \mathrm{II}} f_{j}^{0}\left(z_{i}\right) \text { if } z_{i} \in \mathrm{II}
\end{array}\right.
$$

where:

$$
\left\{\begin{array}{l}
V^{\mathrm{I}, \mathrm{II}}=\left(B^{\mathrm{II}} A^{\mathrm{II}}-\mathbf{A} \overline{A^{\mathrm{I}}}-\overline{B^{\mathrm{I}}}\right)^{-1}\left(B^{\mathrm{I}}-B^{\mathrm{II}} A^{\mathrm{II}}{ }^{-1} A^{\mathrm{I}}\right) \\
W^{\mathrm{I}, \mathrm{II}}=\left(\overline{B^{\mathrm{I}} A^{\mathrm{I}^{-1}}} A^{\mathrm{II}}-B^{\mathrm{II}}\right)^{-1}\left(\overline{B^{\mathrm{I}} A^{\mathrm{I}^{-1}}} A^{\mathrm{I}}-B^{\mathrm{I}}\right)
\end{array}\right.
$$

Displacement and stress fields are then deduced from Eqs. ( 5 ), ( 6 ), (12 ), ( 13 ) and ( 14 ). It is noteworthy that when $z_{i} \in \mathrm{II}, f_{j}^{0}\left(z_{i}\right)=f_{j}^{0}\left(z_{i}^{\mathrm{II}}\right)=q_{j}^{0} \ln \left(z_{i}^{\mathrm{II}}-s_{j}^{\mathrm{I}}\right)$ with $s_{j}^{\mathrm{I}}=X_{1}+p_{j}^{\mathrm{I}} X_{2}$ since $\boldsymbol{f}^{\mathbf{0}}$ is associated to material I (just as $\boldsymbol{q}^{\mathbf{0}}$ ). In the case where the dislocation is assumed to be located in the lower material II, the solution is obtained by a similar procedure yielding:

$$
\begin{aligned}
& f_{i}\left(z_{i}\right)=\left\{\begin{array}{c}
f_{i}^{\mathrm{I}}\left(z_{i}\right) \quad \text { if } z_{i} \in \mathrm{I} \\
f_{i}^{0}\left(z_{i}\right)+f_{i}^{\mathrm{II}}\left(z_{i}\right) \quad \text { if } z_{i} \in \mathrm{II}
\end{array}\right. \\
& \begin{cases}f_{i}^{\mathrm{I}}\left(z_{i}^{\mathrm{I}}\right)=W_{i j}^{\mathrm{II}, \mathrm{I}} f_{j}^{0}\left(z_{i}\right) & \text { if } z_{i} \in \mathrm{I} \\
f_{i}^{\mathrm{II}}\left(z_{i}^{\mathrm{II}}\right)=\overline{V_{i j}^{\mathrm{II}, \mathrm{I}}} \frac{f_{\underline{j}}^{0}}{}\left(z_{i}\right) & \text { if } z_{i} \in \mathrm{II}\end{cases}
\end{aligned}
$$

where $f_{j}^{0}\left(z_{i}\right)$ is still calculated from Eq. ( 10$)$ but now considering the stiffness tensor $\boldsymbol{C}^{\mathbf{I I}}$ of the lower material II and $s_{j}=s_{j}^{\mathrm{II}}=X_{1}+p_{j}^{\mathrm{II}} X_{2}$. 


\subsection{Half-space with rigid or free surface}

The problem of a singularity in a half-space I with a surface at $x_{2}=0$ can be solved in a similar manner as the bi-material problem (Suo, 1990; Hwu, 2010). If the surface is assumed to be rigid, the displacement boundary condition is $u_{i}^{\mathrm{I}}\left(x_{2}=0\right)=0$. Displacement and stress fields are then obtained by considering Eqs. ( 5 ), ( 6 ), along with $f_{i}\left(z_{i}\right)=f_{i}^{0}\left(z_{i}\right)+f_{i}^{\mathrm{I}}\left(z_{i}\right)$ where:

$$
f_{i}^{\mathrm{I}}\left(z_{i}\right)=-A_{i k}^{I-1} \overline{A_{k j}^{I}} \overline{f_{\underline{j}}^{0}}\left(z_{i}\right)
$$

If the surface is assumed to be traction-free, the continuity of the resultant traction force becomes $\phi_{i}^{\mathrm{I}}\left(x_{2}=0\right)=0$ and the expression of $f_{i}^{\mathrm{I}}\left(z_{i}\right)$ is:

$$
f_{i}^{\mathrm{I}}\left(z_{i}\right)=-B_{i k}^{I-1} \overline{B_{k j}^{I}} \overline{f_{\underline{j}}^{0}}\left(z_{i}\right)
$$

\section{Heterogeneous medium: tri-material}

\subsection{Configuration of tri-material}

In section 4.2 , the bi-material was regarded as the combination of two materials perfectly bonded to each other through an interface without thickness. In the case where the considered interface is a grain boundary (GB), the real thickness is finite. At a suitable resolution scale, it may be thus interesting to investigate the effects of the GB thickness and stiffness on the elastic fields in a bi-crystal considered as a tri-material. As discussed in the introduction, several studies were concerned with multilayered anisotropic elastic media. In the present paper, the method of Choi and Earmme (2002a) is followed.

Figure 2 shows the considered tri-material configuration. There are two planar interfaces $\Gamma_{1}$ and $\Gamma_{2}$ whose normals are directed along the $x_{2}$ axis. These interfaces are located, respectively, at $x_{2}=h$ and at $x_{2}=-d$. The interphase (or grain boundary) thickness is thus $H=h+d$. Material I corresponds to $x_{2} \geq h$, material II to $-d<x_{2}<h$ and material III to $x_{2} \leq-d$. All the materials are assumed to be perfectly bonded to each other. 


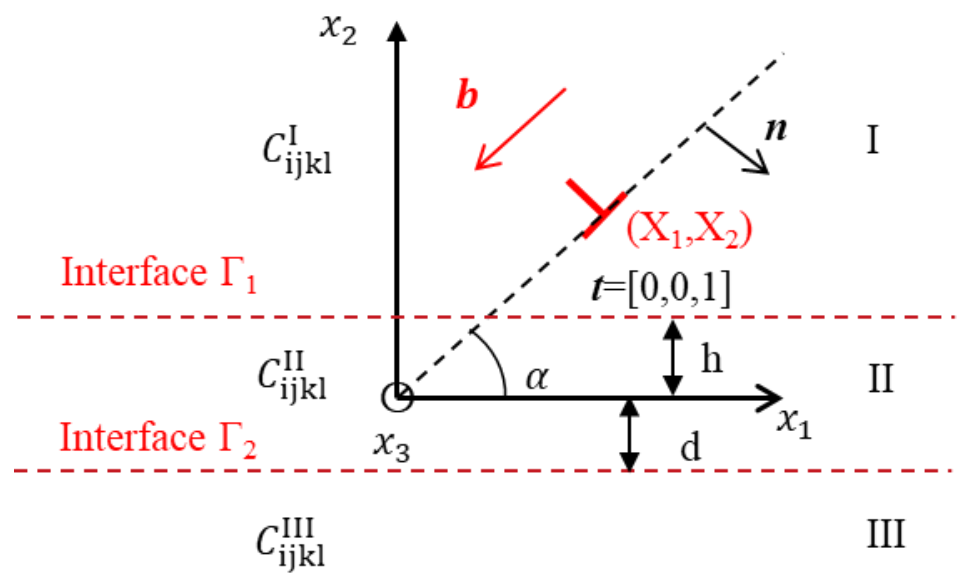

Figure 2. A single straight edge dislocation in a tri-material with three different stiffness tensors and two perfectly bonded interfaces. The grain boundary region is supposed to be material II.

\subsection{A coordinate translation}

In contrast with section 4.2 , it is noteworthy that the interfaces are no more located at $x_{2}=0$. Hence, a coordinate translation should be performed first in order to be able to use correctly the bi-material expressions of elastic fields. Let us then consider a bi-material configuration where the dislocation is located in the upper crystal I and where the interface is located at $x_{2}=h>0$. In this case, the change of coordinates $x_{2} \rightarrow x_{2}-h$ and $X_{2} \rightarrow X_{2}-h$ must be performed in order to retrieve the same bi-material configuration as the one of section 4.2, i.e. such that the interface would be at $x_{2}=0$ within the new coordinate system. Hence, $z$ and $s$ must be modified as $z_{i} \rightarrow z_{i}-h p_{i}^{\mathrm{I}}$ and $s_{j} \rightarrow s_{j}-h p_{j}^{\mathrm{I}}$, which leads to the following change of coordinates for $f_{j}^{0}\left(z_{i}\right)$ (Choi and Earmme, 2002a): 


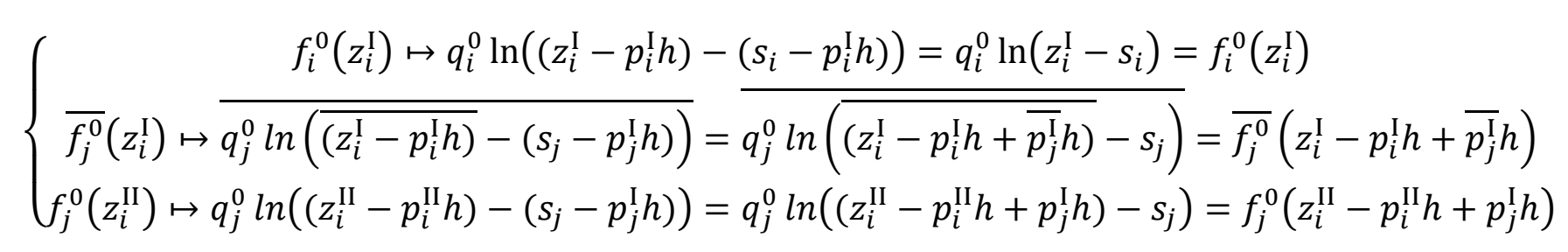

It can be observed that the expression of $f_{i}^{0}\left(z_{i}^{\mathrm{I}}\right)$ remains unchanged since it corresponds to the case of a dislocation in a homogeneous anisotropic material (no interface). However, the two other terms have been modified because they depend on the position of the interface. The solutions for a bi-material with the interface located at $x_{2}=h>0$ are then deduced from Eqs. ( 5 ), ( 6 ), (12 ) and ( 13 ) along with:

$$
\left\{\begin{array}{c}
f_{i}^{\mathrm{I}}\left(z_{i}^{\mathrm{I}}\right)=\overline{V_{i \underline{j}}^{\mathrm{IIII}}} \overline{f_{\underline{j}}^{0}}\left(z_{i}^{\mathrm{I}}-p_{i}^{\mathrm{I}} h+\overline{p_{\underline{j}}^{\mathrm{I}}} h\right) \\
f_{i}^{\mathrm{II}}\left(z_{i}^{\mathrm{II}}\right)=W_{i \underline{j}}^{\mathrm{III}} f_{\underline{j}}^{0}\left(z_{i}^{\mathrm{II}}-p_{i}^{\mathrm{II}} h+p_{\underline{j}}^{\mathrm{I}} h\right)
\end{array}\right.
$$

Thanks to a similar procedure, the solutions for the different cases displayed in Table 1 are also derived, i.e. when the dislocation is located in the lower crystal II or when the interface is

\begin{tabular}{|c|c|c|}
\hline & Interface $x_{2}=h>0$ & Interface $x_{2}=-d<0$ \\
\hline $\begin{array}{l}\text { Dislocation } \\
\text { in upper } \\
\text { crystal I }\end{array}$ & $\begin{array}{l}f_{i}^{\mathrm{I}}\left(z_{i}\right)=\overline{V_{i \underline{j}}^{\mathrm{I}, \mathrm{II}}} \overline{f_{j}^{0}}\left(z_{i}-p_{i}^{\mathrm{I}} h+\overline{p_{\underline{j}}^{\mathrm{I}}} h\right) \\
f_{i}^{\mathrm{II}}\left(z_{i}\right)=W_{i \underline{j}}^{\mathrm{III}} f_{\underline{j}}^{0}\left(z_{i}-p_{i}^{\mathrm{II}} h+p_{\underline{j}}^{\mathrm{I}} h\right)\end{array}$ & $\begin{aligned} f_{i}^{\mathrm{I}}\left(z_{i}\right) & =\overline{V_{i \underline{j}}^{\mathrm{I}, \mathrm{II}}} \overline{f_{j}^{0}}\left(z_{i}+p_{i}^{\mathrm{I}} d-\overline{p_{\underline{j}}^{\mathrm{I}}} d\right) \\
f_{i}^{\mathrm{II}}\left(z_{i}\right) & =W_{i \underline{j}}^{\mathrm{III}} f_{\underline{j}}^{0}\left(z_{i}+p_{i}^{\mathrm{II}} d-p_{\underline{j}}^{\mathrm{I}} d\right)\end{aligned}$ \\
\hline $\begin{array}{l}\text { Dislocation } \\
\text { in lower } \\
\text { crystal II }\end{array}$ & $\begin{array}{l}f_{i}^{\mathrm{I}}\left(z_{i}\right)=W_{i \underline{\underline{I}}}^{\mathrm{II}, \mathrm{I}} f_{\underline{j}}^{0}\left(z_{i}-p_{i}^{\mathrm{I}} h+p_{\underline{j}}^{\mathrm{II}} h\right) \\
f_{i}^{\mathrm{II}}\left(z_{i}\right)=\overline{V_{i \underline{j}}^{\mathrm{II}, \mathrm{I}}} \overline{f_{\underline{j}}^{0}}\left(z_{i}-p_{i}^{\mathrm{II}} h+\overline{p_{\underline{j}}^{\mathrm{II}}} h\right)\end{array}$ & $\begin{array}{l}f_{i}^{\mathrm{I}}\left(z_{i}\right)=W_{i \underline{j}}^{\mathrm{II}, \mathrm{I}} f_{\underline{j}}^{0}\left(z_{i}+p_{i}^{\mathrm{I}} d-p_{\underline{j}}^{\mathrm{II}} d\right) \\
f_{i}^{\mathrm{II}}\left(z_{i}\right)=\overline{V_{i \underline{j}}^{\mathrm{II}, \mathrm{I}}} \overline{f_{\underline{j}}^{0}}\left(z_{i}+p_{i}^{\mathrm{II}} d-\overline{p_{\underline{j}}^{\mathrm{II}}} d\right)\end{array}$ \\
\hline
\end{tabular}
located at $x_{2}=-d<0$.

Table 1 Bi-material solution functions for different locations of the interface and dislocation. 


\subsection{Alternating technique with standard analytic continuation arguments}

In the tri-material configuration, there are two interfaces and the difficulty is thus to satisfy the continuity of displacements and forces across the two interfaces at the same time. Choi and Earmme (2002a) overcame this difficulty by applying the alternating technique, which consists in satisfying alternatively the continuity conditions across each interface until a convergence is obtained. That means that both materials which are on the same side of the considered interface are regarded as a homogeneous material. The latter corresponds to the material adjacent to the interface. At each step $\beta$, the function which is used as a homogeneous solution to perform the computation is updated (see details in (Choi and Earmme, 2002a)).

Still considering the case of a dislocation located in the crystal I and by using the same procedure as the one of Choi and Earmme (2002a), the following series solution is obtained:

$$
f_{i}\left(z_{i}\right)=\left\{\begin{array}{lr}
f_{i}^{0}\left(z_{i}\right)+f_{i}^{\mathrm{I} 0}\left(z_{i}\right)+\sum_{\beta=1}^{\infty} f_{i}^{\mathrm{I} \beta}\left(z_{i}\right) & \text { if } z_{i} \in \mathrm{I} \\
\sum_{\beta=1}^{\infty} f_{i}^{\beta}\left(z_{i}\right)+\sum_{\beta=1}^{\infty} f_{i}^{\mathrm{II} \beta}\left(z_{i}\right) & \text { if } z_{i} \in \mathrm{II} \\
\sum_{\beta=1}^{\infty} f_{i}^{\mathrm{III} \beta}\left(z_{i}\right) & \text { if } z_{i} \in \mathrm{III}
\end{array}\right.
$$

where:

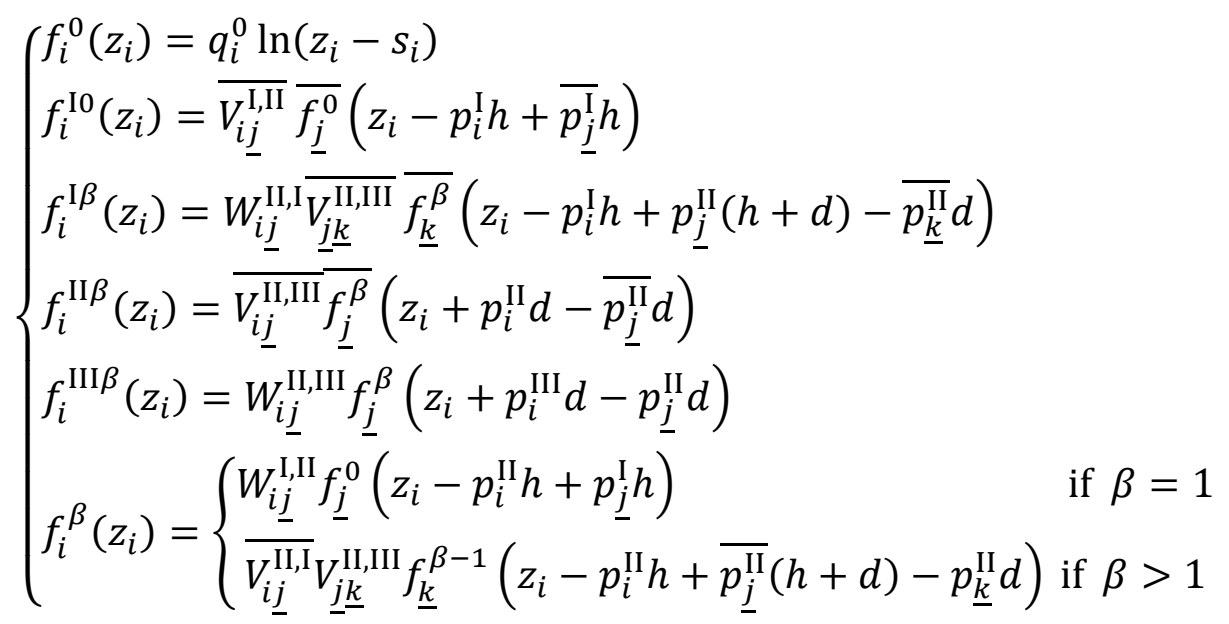

In the above equations, $f_{j}^{0}\left(z_{i}\right)$ is associated to crystal I and so $s_{j}=s_{j}^{\mathrm{I}}=X_{1}+p_{j}^{\mathrm{I}} X_{2}$. The series solution $f_{i}^{\mathrm{I} \beta}\left(z_{i}\right), f_{i}^{\mathrm{II} \beta}\left(z_{i}\right)$ and $f_{i}^{\mathrm{III} \beta}\left(z_{i}\right)$ are all expressed as functions of $f_{j}^{\beta}\left(z_{i}\right)$ which is itself determined by a recurrence equation based on $f_{j}^{0}\left(z_{i}\right)$. 
In a similar manner, when the dislocation is located in the crystal II (interphase, i.e. GB region), the series solution is:

$$
f_{i}\left(z_{i}\right)=\left\{\begin{array}{l}
\sum_{\beta=1}^{\infty} f_{i}^{\mathrm{I} \beta}\left(z_{i}\right) \quad \text { if } z_{i} \in \mathrm{I} \\
\sum_{\beta=1}^{\infty} f_{i}^{\beta}\left(z_{i}\right)+\sum_{\beta=1}^{\infty} f_{i}^{\mathrm{II} \beta}\left(z_{i}\right) \quad \text { if } z_{i} \in \mathrm{II} \\
f_{i}^{\mathrm{III} 0}\left(z_{i}\right)+\sum_{\beta=1}^{\infty} f_{i}^{\mathrm{II} \beta}\left(z_{i}\right) \quad \text { if } z_{i} \in \mathrm{III}
\end{array}\right.
$$

where:

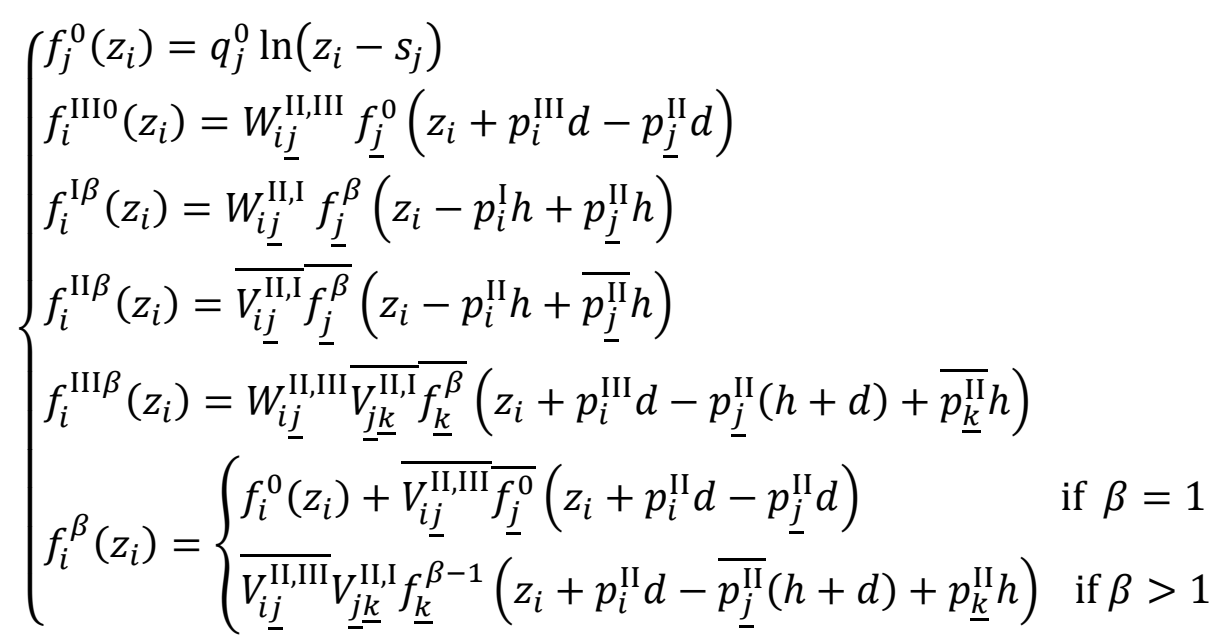

In the above equations, $f_{j}^{0}\left(z_{i}\right)$ is now associated to crystal II and so $s_{j}=s_{j}^{\mathrm{II}}=X_{1}+p_{j}^{\mathrm{II}} X_{2}$.

\subsection{Convergence of series solution through three criteria}

Choi and Earmme (2002a) proved that the above series solutions are indeed convergent (see the discussion in the section 6 of their paper). However, for numerical applications, it is important to discuss the definition of different convergence criteria in order to analyze the rate of convergence of the series. When the truncation of the series $\beta$ becomes large, the computation is very time-consuming or even unrealizable for large values of mechanical contrasts between crystals. To study numerical convergence, Choi and Earmme (2002a) considered a criterion based on the image force acting on the dislocation and discussed the effect of the elastic stiffness of the different materials as well as the one of the thickness of the second material. Their criterion is expressed as: 


$$
\epsilon_{F}^{\beta+1}=\frac{\left|F_{i m}^{\beta+1}-F_{i m}^{\beta}\right|}{\left|F_{i m}^{\beta+1}\right|}
$$

where $F_{i m}^{\beta+1}$ is the component of the image force along the $x_{2}$ axis at the position of the dislocation. They considered that convergence was achieved for $\epsilon_{F}^{\beta+1}<10^{-4}$. This criterion is based only on stress and disregards the solutions outside the dislocation, in particular in the other materials.

In the present paper, two new convergence criteria are developed. The first one considers the norm of the displacement vector difference between two steps summed at every point $(\mathrm{P}, \mathrm{Q})$ of the simulation plane (which includes the three materials):

$$
\epsilon_{u}^{\beta+1}=\frac{\sum_{P, Q} \sqrt{\sum_{i}\left[u_{i}^{\beta+1}(P, Q)-u_{i}^{\beta}(P, Q)\right]^{2}}}{\sum_{P, Q} \sqrt{\sum_{i}\left[u_{i}^{\beta+1}(P, Q)\right]^{2}}}
$$

The second one considers the norm of the stress tensor difference between two steps summed at every point $(\mathrm{P}, \mathrm{Q})$ of the simulation plane:

$$
\epsilon_{\sigma}^{\beta+1}=\frac{\sum_{P, Q} \sqrt{\sum_{i j}\left[\sigma_{i j}^{\beta+1}(P, Q)-\sigma_{i j}^{\beta}(P, Q)\right]^{2}}}{\sum_{P, Q} \sqrt{\sum_{i j}\left[\sigma_{i j}^{\beta+1}(P, Q)\right]^{2}}}
$$

Through the combination of these two criteria, both displacement and stress distributions are considered. Contrary to the criterion of Choi and Earmme (2002a) which focuses on only one material point, the above criteria depends on the size and the spatial discretization (mesh resolution) of the simulation plane. Indeed, it can be soundly inferred that the elastic field differences between two steps are larger close to the singularity than far away. However, the numerical calculations reported in Section 7.2 will show that the influence of the simulation plane's characteristics was negligible.

\section{Discrete dislocation pile-up theory}

6.1 Determination of dislocation pile-up equilibrium positions in heterogeneous bi-materials, tri-materials and half spaces

Let us consider a single dislocation pile-up of $\mathrm{N}$ infinite straight edge dislocations, which are 
all parallel to the $x_{3}$ axis, have the same Burgers $\boldsymbol{b}$ and lie in the same slip plane as shown in Figure 3.

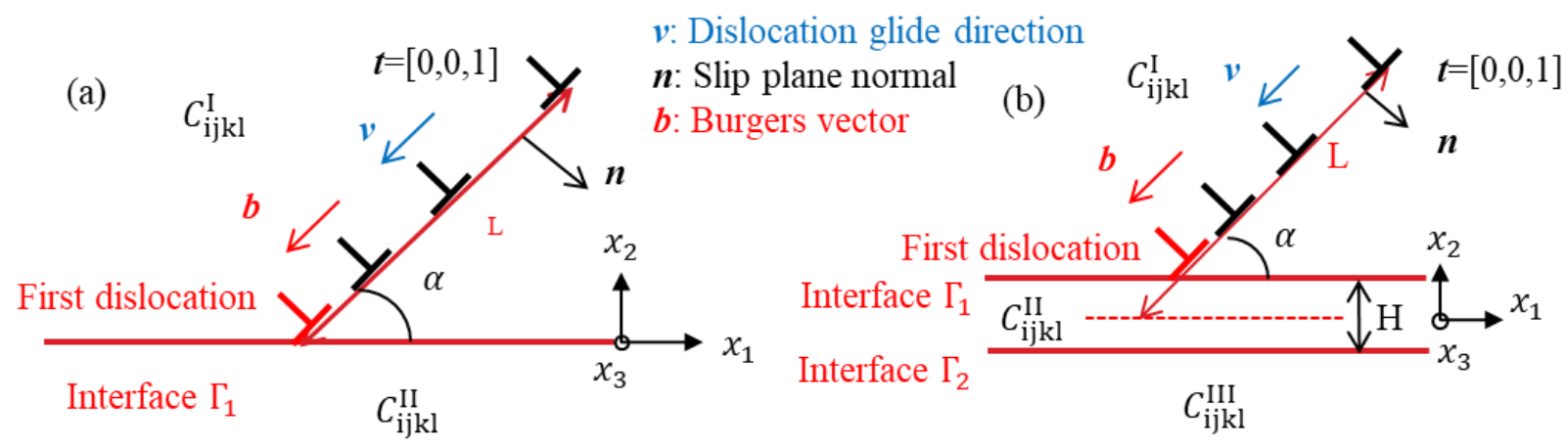

Figure 3. $\alpha$-inclined dislocation pile-up in slip plane of unit normal $\mathbf{n}$ for $\mathbf{N}$ edge dislocations with Burgers vector $\mathbf{b}$ and line vector $\boldsymbol{t}$ in heterogeneous anisotropic (a) bi-material and (b) tri-material.

The equilibrium positions of the $\mathrm{N}$ dislocations can be found out by minimizing to zero the component of the Peach-Koehler (P-K) (Peach and Koehler, 1950) force along the slip direction for each dislocation (Eshelby et al., 1951). In practice, a value less than $5 \times 10^{-6} \mathrm{~N} / \mathrm{m}$ is used as in Wagoner (1981). Hereafter, the computation of the P-K force follows the Final-Start, Right-Hand (FSRH) convention (see Kelly and Groves, 1970). This means that, for a non-screw dislocation (here edge dislocations), the positive normal to the slip plane is given by $\boldsymbol{n}=\boldsymbol{t} \times \boldsymbol{b} /|\boldsymbol{b}|$ where $\boldsymbol{t}$ is the unit line direction (cf. Figure 3). The projection of the $\mathrm{P}-\mathrm{K}$ force of the $\gamma^{\text {th }}$ dislocation $(\gamma=1, \ldots, \mathrm{N})$ along the glide direction is computed as follows:

$$
F^{(\gamma)}=\left\{\left(\boldsymbol{\sigma}_{\text {int }}\left(X_{1}(\gamma), X_{2}(\gamma)\right)+\boldsymbol{\sigma}_{\text {ext }}\right) \cdot \boldsymbol{b} \times \boldsymbol{t}\right\} \cdot \boldsymbol{v}
$$

where $\left(X_{1}(\gamma), X_{2}(\gamma)\right)$ denotes the position of the $\gamma^{\text {th }}$ dislocation. $v$ is a unit vector indicating the glide direction of all dislocations, which is considered to be directed towards the interface so that a pile-up can form. Accordingly, $F^{(\gamma)}>0$ means that the $\gamma^{\text {th }}$ dislocation is attracted by the grain boundary (attractive GB) whereas $F^{(\gamma)}<0$ means that the $\gamma^{\text {th }}$ dislocation is repelled by the grain boundary (repulsive GB). $v$ belongs to the slip plane and is normal to the dislocation line. For edge dislocations as considered in the present paper, $\boldsymbol{v}$ is collinear to the slip direction. $\boldsymbol{\sigma}_{\text {ext }}$ is a homogenous applied stress tensor and 
$\boldsymbol{\sigma}_{\text {int }}$ is the internal stress tensor produced by all the dislocations. It should be pointed out that in the present work, the introduction of the homogenous stress tensor $\boldsymbol{\sigma}_{\text {ext }}$ has only for objective to equilibrate the dislocation pile-up. Even in the absence of singularities like dislocations, an infinite heterogeneously elastic material that is submitted to some (remotely) applied stress will actually display stress heterogeneities due to incompatibility stresses that are needed to maintain the continuity conditions at the interfaces (Gemperlová et al., 1989; Richeton and Berbenni, 2013; Tiba et al., 2015). In this paper, these long-range incompatibility stresses due to heterogeneous anisotropic elasticity are neglected since only the normal 22-component of $\boldsymbol{\sigma}_{\text {ext }}$ is non-zero. From traction continuity condition at an interface with normal along $\boldsymbol{e}_{2}$, this component is uniform in the infinite material, so this one does not exhibit incompatibility stress, but other incompatibility stress 11, 33 and 13 components may be present. It is here conjectured that these ones are small compared to the applied stress field. In linear elasticity, $\sigma_{\text {int }}$ is computed as the superposition of the stress tensor of each individual dislocation, which is obtained from the expressions derived in Sections 4 and 5. In an infinite homogenous medium, the self-contribution of the $\gamma^{\text {th }}$ dislocation at its position is considered to be zero because of the dislocation self-equilibrium. In an heterogeneous medium (half-space, bi- or tri-material, cf. Figure 3), the contribution of the image force due to the presence of interface(s) or surface should be considered for all the dislocations in $\sigma_{\text {int }}$, i.e. only the self-contribution related to the homogeneous function $f_{i}^{0}\left(z_{i}\right)$ is omitted (cf. Eqs. (12), ( 13 ) and ( 14$)$ ).

The calculation of the dislocation pile-up equilibrium positions is thus obtained by following an iterative relaxation scheme that minimizes all the $F^{(\gamma)}$ after an initial configuration is specified. In order to be able to perform such a computation in an infinite homogenous medium, one dislocation should be considered as locked. Usually, the position of the first (or leading) dislocation $\left(X_{1}(1), X_{2}(1)\right)$ is fixed (Mitchell et al., 1965, Wagoner, 1981). As a result, the P-K force on the locked dislocation is not zero. In an heterogeneous medium, the boundary image force on the leading dislocation may equilibrate the applied stress and the stress contribution coming from the other dislocations (Wagoner, 1981), but only in case of 
repulsive image force. In such case, all the dislocation positions, including the leading one, could be found by the iterative relaxation scheme.

In the present paper, the case where the position of the leading dislocation is locked is studied in section 7.5 in order to perform consistent comparisons of pile-up lengths between different configurations such as homogeneous material, heterogeneous bi-material (Figure 3 (a)), half-space (Figure 3 (a)) and heterogeneous tri-material (Figure 3 (b)). The case where the leading dislocation is unlocked is studied in section 7.6

\subsection{Resolved shear stress in front of dislocation pile-ups}

According to the theoretical work of Eshelby et al. (1951) performed in isotropic elasticity, a lower bound estimate of the resolved shear stress on the slip system of a pile-up of straight dislocations is:

$$
\tau=\tau_{0} \sqrt{\frac{L}{d}}=\sqrt{2 A \tau_{0}} \sqrt{\frac{N}{d}} \text { for } \frac{1}{15} l \ll d \ll L
$$

where $d$ is the distance beyond the leading (locked) dislocation, $\tau_{0}$ the applied resolved shear stress, $L$ the pile-up length and $l$ the distance between the locked and the nearest free dislocation as shown in Figure 4. For a large number of dislocations in isotropic elasticity, $L$ can be approximated by $L=\frac{2 N A}{\tau_{0}}$ with $A=\frac{G|\boldsymbol{b}|}{2 \pi(1-v)}$ for edge dislocations (Eshelby et al., 1951). 


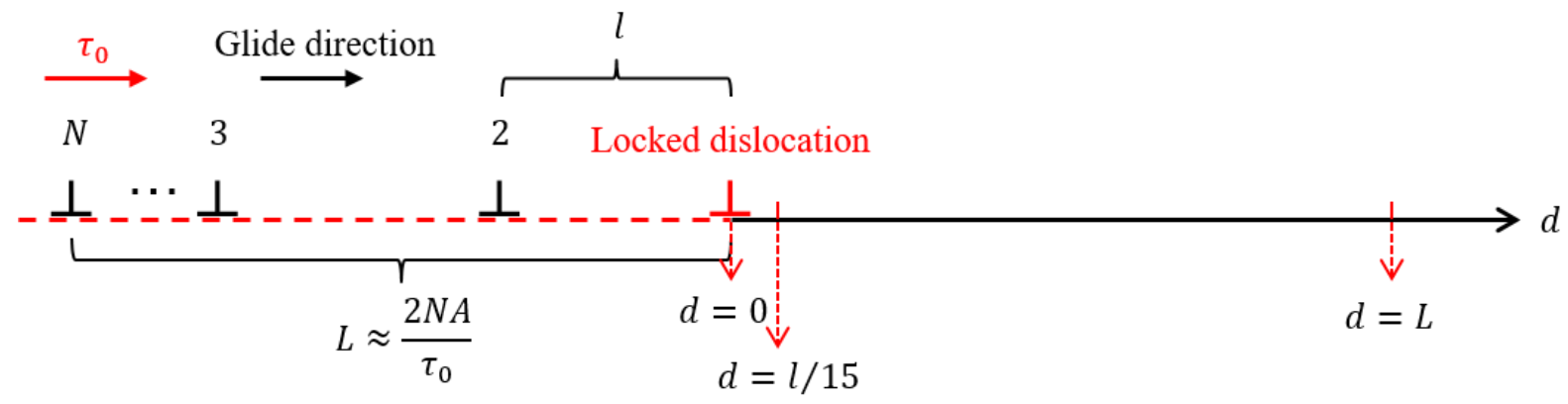

Figure 4. Discrete edge dislocation pile-up configuration in isotropic elasticity (Eshelby et al., 1951).

\section{Results and discussion}

\subsection{Different studied configurations}

In all the following numerical applications, nickel $(\mathrm{Ni})$ bi-crystalline configurations are considered, i.e. two crystals of $\mathrm{Ni}$, which can have different crystallographic orientations. In case of a tri-material configuration, the interphase material is supposed to represent the grain boundary (GB) region. Ni has a face centered cubic (FCC) structure and was chosen due to its moderate elastic anisotropy (Zener coefficient $\left.A_{a n i}=2 \mathrm{C}_{44} /\left(\mathrm{C}_{11}-\mathrm{C}_{12}\right)=2.51\right)$. The elastic stiffness values are presented in Table 2 . The role of crystal plasticity on slip incompatibilities in Ni polycrystals and bi-crystals were recently experimentally and theoretically studied in Perrin et al. (2010) and Tiba et al. (2015) but without considering discrete pile-up with anisotropic elasticity.

Furthermore, only edge dislocations having their Burgers vector in the $\left(\boldsymbol{e}_{\mathbf{1}}, \boldsymbol{e}_{\mathbf{2}}\right)$ plane are considered. As shown in Figure 1, the Burgers vector can be determined by an angle $\alpha$, so that $\boldsymbol{m}=\boldsymbol{b} /|\boldsymbol{b}|=[-\cos \alpha,-\sin \alpha, 0]$ in the global frame $\left(\boldsymbol{e}_{1}, \boldsymbol{e}_{2}, \boldsymbol{e}_{3}\right)$. Still following the FSRH convention, the slip plane normal is given by $\boldsymbol{n}=\boldsymbol{t} \times \boldsymbol{m}$ where $\boldsymbol{t}=[0,0,1]$. The slip system is supposed to be one of the usual twelve slip systems for FCC crystals, for example $\boldsymbol{m}^{\mathbf{0}}=1 / \sqrt{2}[1,1,0]$ and $\boldsymbol{n}^{\mathbf{0}}=1 / \sqrt{3}[-1,1,-1]$ in the crystal's frame $\left(\boldsymbol{e}_{\mathbf{1}}^{\mathbf{0}}, \boldsymbol{e}_{2}^{\mathbf{0}}, \boldsymbol{e}_{3}^{\mathbf{0}}\right)$. The 
crystallographic orientation of the upper crystal I is thus fixed by the choice of the angle $\alpha$ and is defined by the transformation matrix $\boldsymbol{T}$ such that $[\boldsymbol{m}, \boldsymbol{n}, \boldsymbol{t}]^{\mathrm{T}}=\boldsymbol{T}\left[\boldsymbol{m}^{\mathbf{0}}, \boldsymbol{n}^{\mathbf{0}}, \boldsymbol{t}^{\mathbf{0}}\right]^{\mathrm{T}}$, where $\boldsymbol{t}^{\mathbf{0}}=\boldsymbol{m}^{\mathbf{0}} \times \boldsymbol{n}^{\mathbf{0}}$. The elastic stiffness tensor in the global frame is then deduced from the transformation matrix $\boldsymbol{T}$ by $C_{i j k l}=T_{i g} T_{j h} C_{g h m n}^{0} T_{k m} T_{l n}$ where $\mathbf{C}^{\mathbf{0}}$ is the elastic stiffness tensor defined in the crystal's frame. In the lower crystal, there is no dislocation and thus the choice of its crystallographic orientation is free. Two specific orientations are considered in the following: orientation $\mathrm{A}^{\text {Ori }}$ with $\boldsymbol{e}_{\mathbf{1}}=\boldsymbol{e}_{\mathbf{1}}^{\mathbf{0}}, \boldsymbol{e}_{2}=\boldsymbol{e}_{2}^{\mathbf{0}}, \boldsymbol{e}_{3}=\boldsymbol{e}_{3}^{\mathbf{0}}$ and orientation $\mathrm{B}^{\text {Ori }}$ with $\boldsymbol{e}_{1}=-\frac{1}{2} \boldsymbol{e}_{\mathbf{1}}^{\mathbf{0}}+\frac{\sqrt{2}}{2} \boldsymbol{e}_{2}^{\mathbf{0}}+\frac{1}{2} \boldsymbol{e}_{3}^{\mathbf{0}}, \boldsymbol{e}_{2}=-\frac{1}{2} \boldsymbol{e}_{\mathbf{1}}^{\mathbf{0}}-\frac{\sqrt{2}}{2} \boldsymbol{e}_{2}^{\mathbf{0}}+\frac{1}{2} \boldsymbol{e}_{3}^{\mathbf{0}}, \boldsymbol{e}_{3}=\frac{\sqrt{2}}{2} \boldsymbol{e}_{\mathbf{1}}^{\mathbf{0}}+\frac{\sqrt{2}}{2} \boldsymbol{e}_{3}^{\mathbf{0}}$. For details, the contracted Voigt notation of elastic stiffness tensor for $\mathrm{Ni}$ with orientation $\mathrm{A}^{\text {Ori }}$ and with orientation $\mathrm{B}^{\mathrm{Ori}}$ are the following:

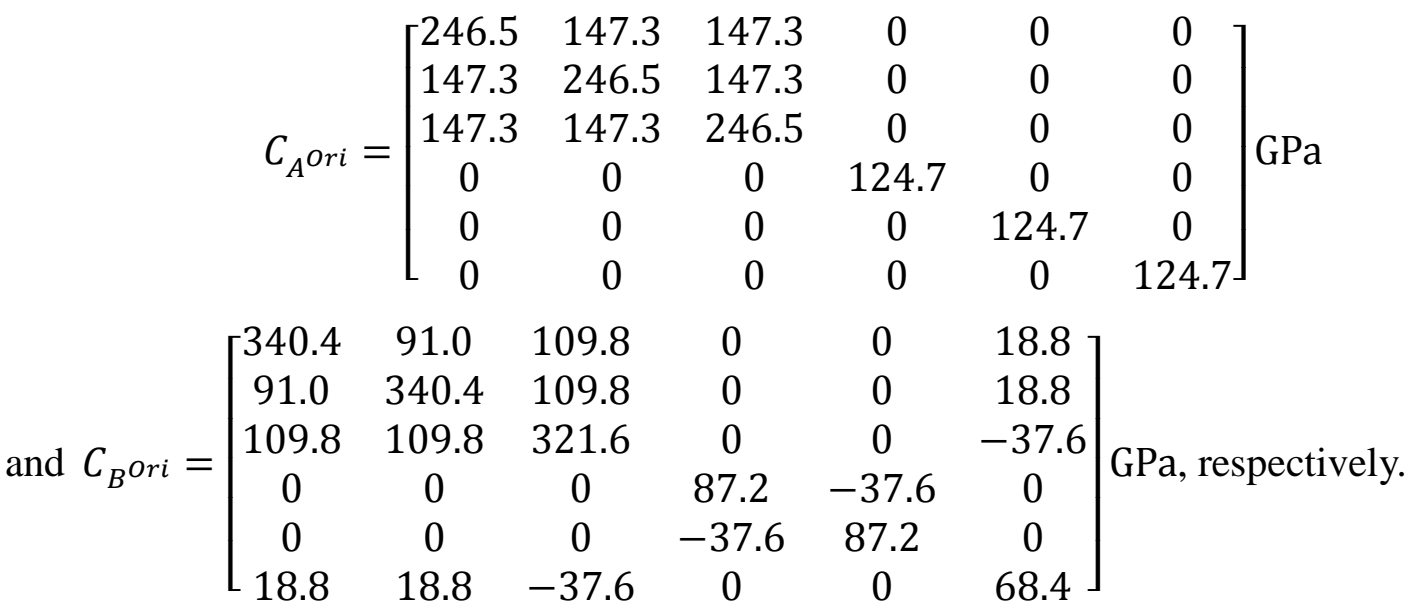

For the tri-material configuration, the thickness of the grain boundary is denoted $H$. GB width should depend on the type of GB and the thermodynamic conditions (Dillon et al., 2007). In metals, the order of magnitude is typically around $1 \mathrm{~nm}$. Hence, a default value of $H=5|\boldsymbol{b}|$ is considered as a first guess in the forthcoming numerical calculations (see Table 2). The GB elastic stiffness tensor $C_{i j k l}^{\mathrm{II}}$ is modeled using a scalar parameter $\lambda$ as:

$$
C_{i j k l}^{\mathrm{II}}=\frac{\lambda}{2}\left(C_{i j k l}^{\mathrm{I}}+C_{i j k l}^{\mathrm{III}}\right)
$$

When $\lambda=1$, the stiffness of the GB corresponds to the average of the two grain stiffness tensors. When $\lambda<1$, the GB is supposed to be softer than the grains. When $\lambda>1$, the grain boundary is supposed to be stiffer than the grains. In the extreme cases, when $\lambda=0$, the 
tri-material reduces to a half-space with a free surface, whereas when $\lambda=+\infty$, the tri-material reduces to a half-space with a rigid surface. Hence, the parameter $\lambda$ allows to study the effect of the GB stiffness on elastic fields of single dislocations and dislocation pile-ups in bi-crystals with GB as a thin interphase region.

The distance between the GB or the middle of the GB region in case of a tri-material configuration and the $\gamma^{\text {th }}$ dislocation along the glide direction is denoted $L(\gamma)$. For dislocation pile-ups, the leading dislocation is locked at $L(1)=5|\boldsymbol{b}|$. The pile-up length is defined as the distance between the first and the last dislocation, i.e. $L(N)-L(1)$.

It is always considered that $\boldsymbol{m}=\boldsymbol{b} /|\boldsymbol{b}|=\boldsymbol{v}$ for edge dislocations and that $0<\alpha<90^{\circ}$ (Burgers vector pointing towards the grain boundary).

For the forthcoming results, a Matlab code was developed to compute the elastic fields over a regular grid of $\mathrm{M}_{x} \times \mathrm{M}_{y}$ points and of $L_{x} \times L_{y}$ in size. If not specifically stated in the text, the values used for numerical applications are the default values reported in Table 2.

\begin{tabular}{cccccc}
\hline $\boldsymbol{C}_{\mathbf{1 1}}(\mathbf{G P a})$ & $\boldsymbol{C}_{\mathbf{1 2}}(\mathbf{G P a})$ & $\boldsymbol{C}_{\mathbf{4 4}}(\mathbf{G P a})$ & $\boldsymbol{G}(\mathbf{G P a})$ & $\boldsymbol{k}(\mathbf{G P a})$ & $|\boldsymbol{b}|(\mathbf{n m})$ \\
\hline 246.5 & 147.3 & 124.7 & 94.7 & 179.8 & 0.25 \\
\hline $\boldsymbol{\sigma}_{\mathbf{2 2}}^{\text {ext }}(\mathbf{M P a})$ & $\boldsymbol{\alpha}\left({ }^{\circ}\right)$ & $\boldsymbol{L}(\mathbf{1})(|\boldsymbol{b}|)$ & \multicolumn{2}{c}{ Orientation of the lower crystal } \\
\hline 100 & 45 & 5 & $\mathrm{~A}^{\text {Ori }}$ \\
\hline $\boldsymbol{\lambda}$ & $\mathbf{H}(|\boldsymbol{b}|)$ & $\boldsymbol{L}_{\boldsymbol{x}} \times \boldsymbol{L}_{\boldsymbol{y}}\left(|\boldsymbol{b}|^{2}\right)$ & $\mathbf{M}_{\boldsymbol{x}} \times \mathbf{M}_{\boldsymbol{y}}$ \\
\hline 1 & 5 & $50 \times 50$ & $100 \times 100$ \\
\hline
\end{tabular}

Table 2. Default values of the parameters used for analytical and numerical simulations.

\subsection{Convergence of the series solutions within the tri-material configuration}

In this sub-section, the three convergence criteria defined and discussed in section $5.4, \epsilon_{F}^{\beta+1}$ 
(Eq. ( 25$)), \epsilon_{u}^{\beta+1}$ (Eq. ( 26$\left.)\right)$ and $\epsilon_{\sigma}^{\beta+1}$ (Eq. ( 27 )) are used to study the influence of three important physical parameters on the convergence of the series solutions in case of a single dislocation within a tri-material configuration (Figure 2). These parameters are the stiffness parameter $\lambda$ (Eq. ( 30$)$ ), the thickness of the interphase $H$ and the distance between the dislocation and the first interface along the glide direction denoted $L_{\Gamma 1}$ (rather than $L(1)$ since $H$ is also varied). To avoid wasting calculation resources, it is indeed important to find out the minimum value of step number $\beta$ allowing the convergence of the series. It is hereafter considered that the convergence is reached when all the three criteria $\epsilon_{F}^{\beta+1}, \epsilon_{u}^{\beta+1}$ and $\epsilon_{\sigma}^{\beta+1}$ are smaller than $10^{-4}$.

First, the effect of $\lambda$ was investigated by setting $H=5|\boldsymbol{b}|, L_{\Gamma 1}=2|\boldsymbol{b}|, \mathrm{M}_{x} \times \mathrm{M}_{y}=200 \times$ 200 and $L_{x} \times L_{y}=200 \times 200|\boldsymbol{b}|^{2}$ while the other parameters are given by the default values presented in Table 2 with lower crystal orientation $A^{\text {Ori }}$. Figure 5 shows the computed errors by the three criteria for $\lambda=0.5,1$ and 2 . It is found that the logarithm of the errors roughly scales with the step number $\beta$. When $\lambda=1$, the convergence is reached for $\beta=3$ while for $\lambda=0.5$ and $\lambda=2$, the convergence is reached for $\beta=5$. It was checked that the convergence becomes more and more difficult when $\lambda$ departs more and more from unity. However, it is noteworthy that if $\lambda$ is zero or is infinite, the model equations of section 5.3 reduces to a half-space with a free or a rigid surface and the exact solutions can be directly obtained from Eqs. ( 17 ) and ( 18 ). 


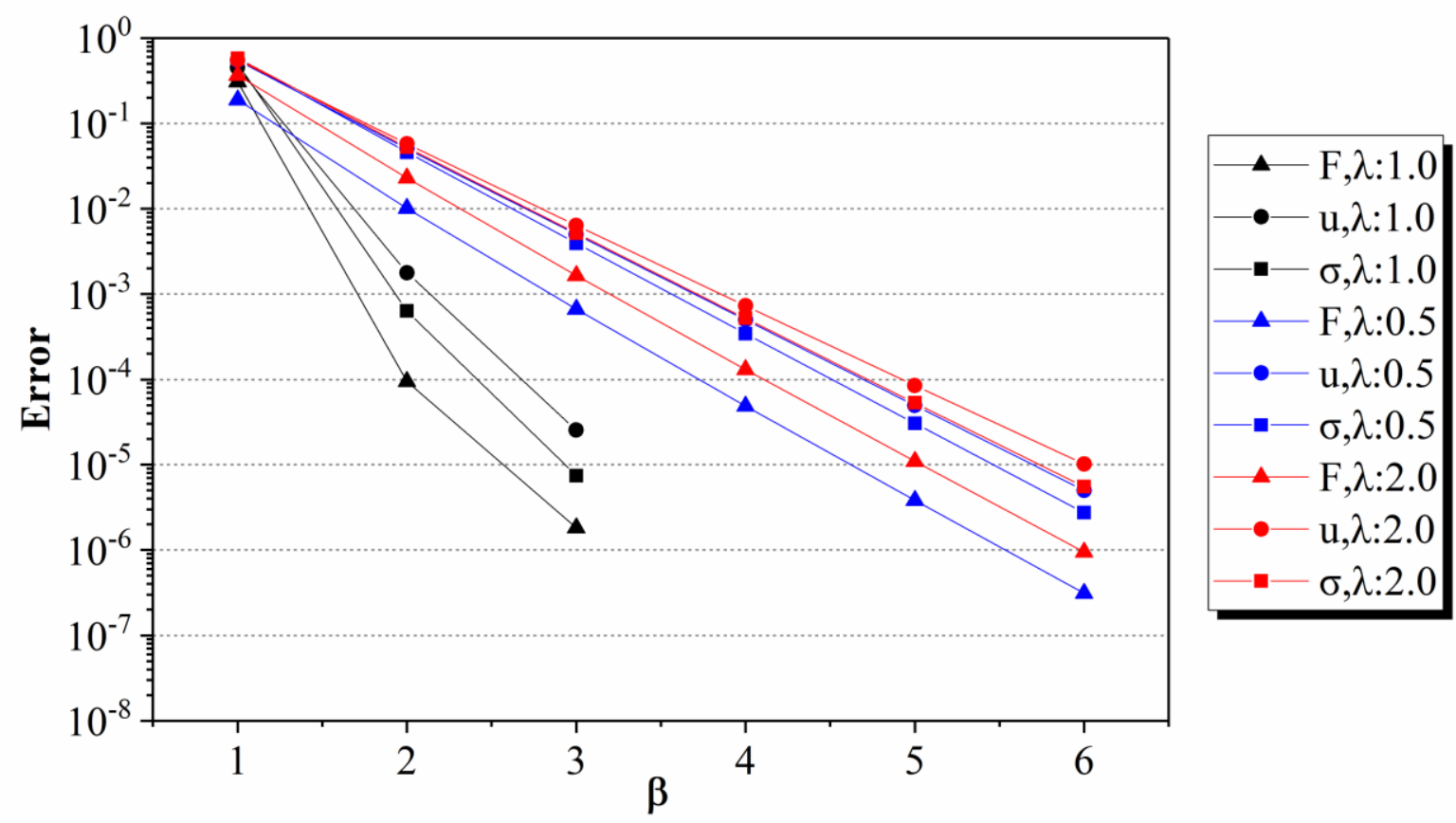

Figure 5. Numerical errors computed by the three criteria $\left(\epsilon_{F}^{\beta+1}, \epsilon_{u}^{\beta+1}, \epsilon_{\sigma}^{\beta+1}\right)$ for $\lambda=0.5,1,2$ (tri-material with lower crystal orientation $\mathrm{A}^{\text {Ori }}$ ).

Second, the effect of $H$ was investigated by setting $\lambda=2, L_{\Gamma 1}=2|\boldsymbol{b}|, \mathrm{M}_{x} \times \mathrm{M}_{y}=200 \times$ 200 and $L_{x} \times L_{y}=200 \times 200|\boldsymbol{b}|^{2}$. Figure 6 shows the numerical errors computed by the three criteria for $H=5|\boldsymbol{b}|, 25|\boldsymbol{b}|$ and $50|\boldsymbol{b}|$. It is found again that the logarithm of the errors scales with the number of steps $\beta$. The error given by $\epsilon_{u}^{\beta+1}$ (dots in Figure 6) is very little dependent on $H$ while it is easier to get convergence on $\epsilon_{F}^{\beta+1}$ and $\epsilon_{\sigma}^{\beta+1}$ when $H$ becomes larger. The reason is that when the thickness of the interphase becomes larger and larger, the tri-material model gets closer and closer to a bi-material configuration and the effects of the lower crystal becomes smaller and smaller. 


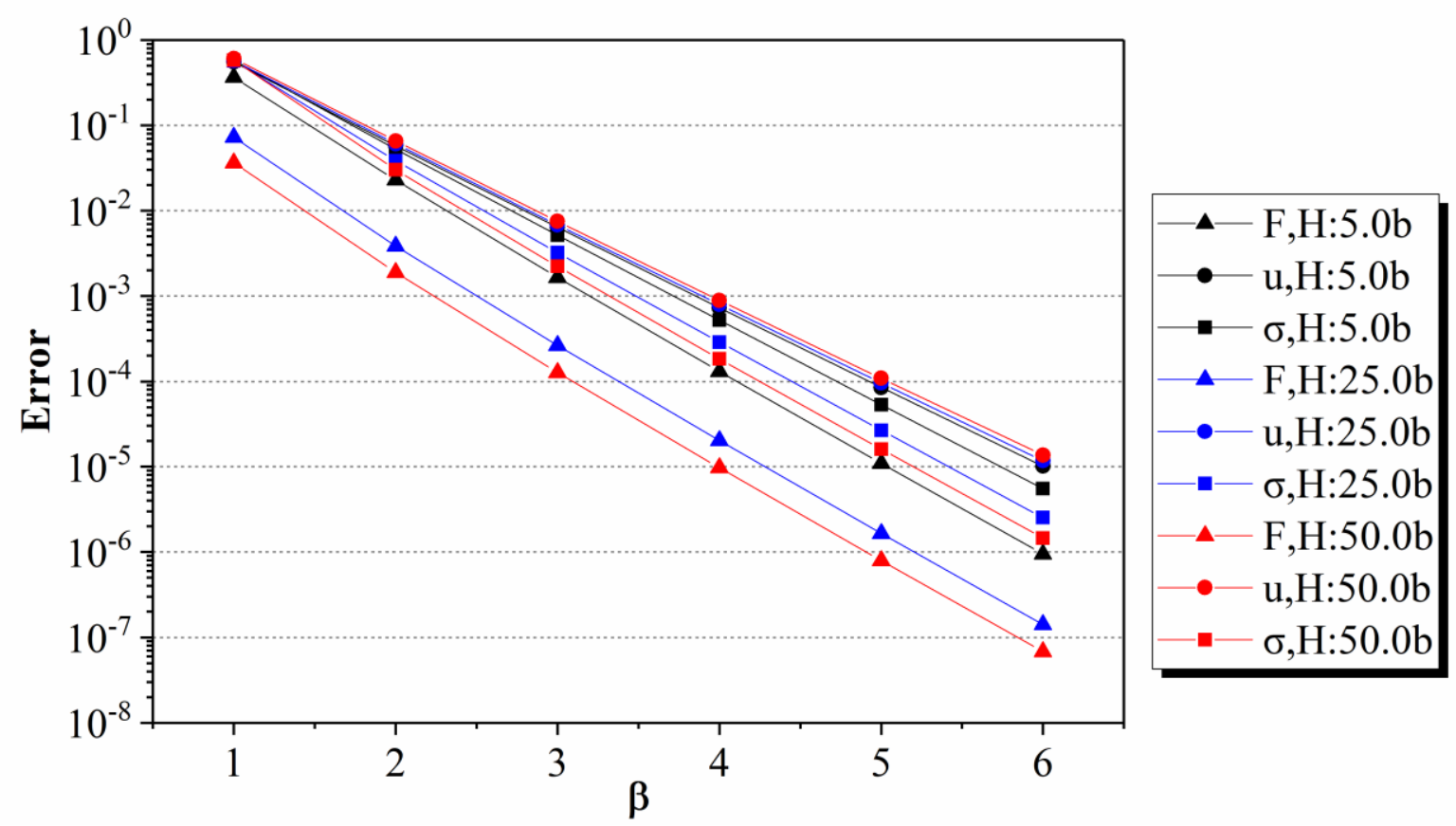

Figure 6. Numerical errors computed by the three criteria $\left(\epsilon_{F}^{\beta+1}, \epsilon_{u}^{\beta+1}, \epsilon_{\sigma}^{\beta+1}\right)$ for $\mathrm{H}=5|\mathbf{b}|$, $25|\mathbf{b}|$ and $50|\mathbf{b}|$ (tri-material with lower crystal orientation $\mathrm{A}^{\mathrm{Ori}}$ ).

Finally, the effect of $L_{\Gamma 1}$ was investigated by setting $\lambda=2, H=5|\boldsymbol{b}|, \mathrm{M}_{x} \times \mathrm{M}_{y}=200 \times$ 200 and $L_{x} \times L_{y}=200 \times 200|\boldsymbol{b}|^{2}$. Figure 7 shows the computed numerical errors by the three criteria for $L_{\Gamma 1}=2|\boldsymbol{b}|, 100|\boldsymbol{b}|$ and $150|\boldsymbol{b}|$. The error given by $\epsilon_{u}^{\beta+1}$ and $\epsilon_{\sigma}^{\beta+1}$ (dots and squares in Figure 7) are very little dependent on $L_{\Gamma 1}$. Contrary to Figure 5 and Figure 6, when $L_{\Gamma 1}=100|\boldsymbol{b}|$ and $150|\boldsymbol{b}|$, the criterion based on $\epsilon_{F}^{\beta+1}$ gives the slowest convergence. The reason is that when the distance between the dislocation and the interface is large, the elastic fields close to the dislocation are weaker than before. Hence, at each step, the relative part of the image force increase is larger compared to the case where the dislocation is close to the interface. 


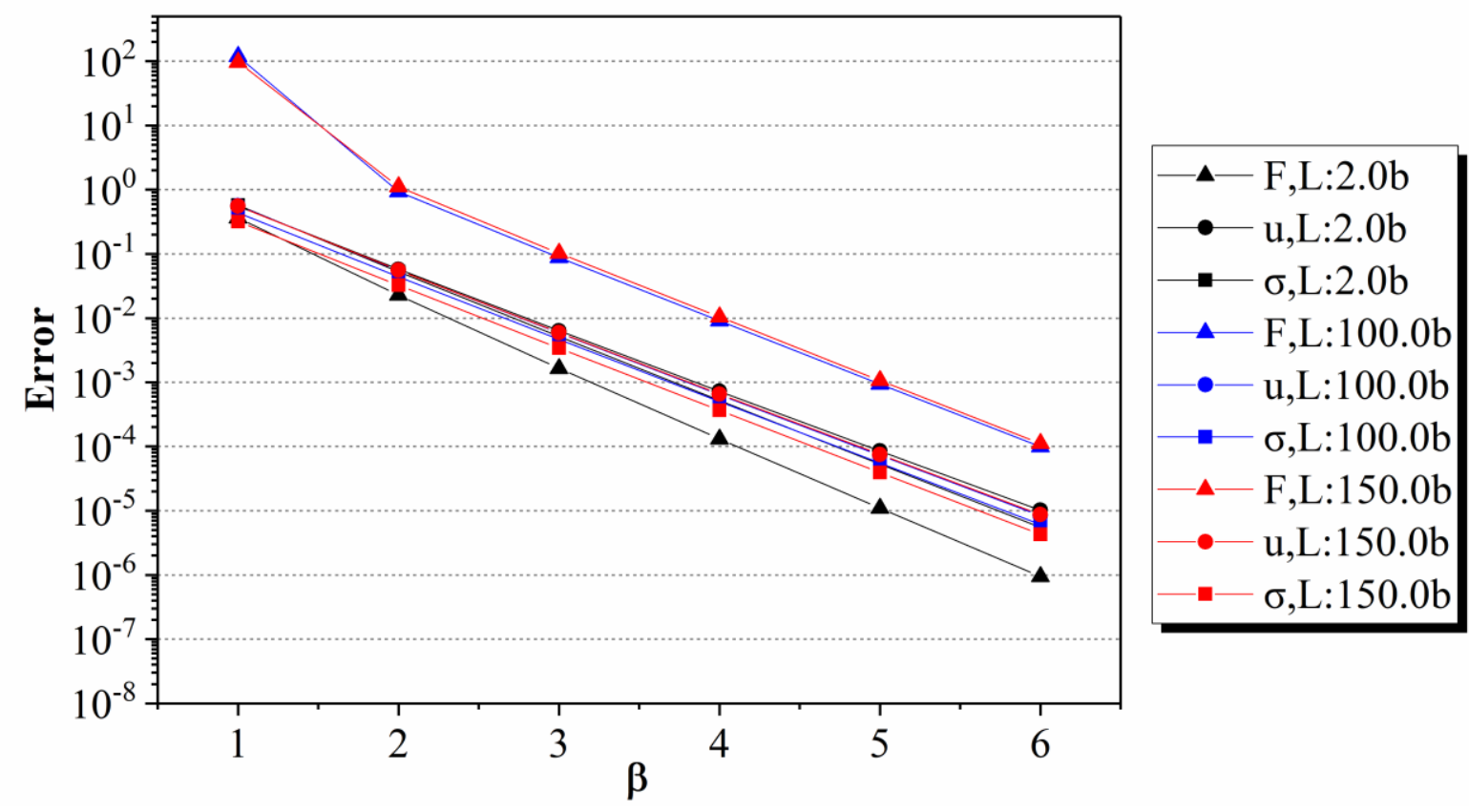

Figure 7. Numerical errors computed by the three criteria $\left(\epsilon_{F}^{\beta+1}, \epsilon_{u}^{\beta+1}, \epsilon_{\sigma}^{\beta+1}\right)$ for $\mathrm{L}_{\Gamma 1}=2|\mathbf{b}|$, $100|\mathbf{b}|$ and $150|\mathbf{b}|$ (tri-material with lower crystal orientation $\mathrm{A}^{\mathrm{Ori}}$ ).

As a consequence of these investigations, the convergence criteria based on $\epsilon_{u}^{\beta+1}$ and $\epsilon_{\sigma}^{\beta+1}$ seem the most appropriate to consider all kinds of situations. Moreover, it was found that only the stiffness parameter $\lambda$ strongly influences the numerical convergence. Hence, for a same value of $\lambda$, a same value of $\beta$ will be considered in the applications.

The obtained values of $\beta$ for different $\lambda$ in case of anisotropic elasticity and isotropic elasticity are presented in Table 3. The computations of the elastic fields in a tri-material with heterogeneous isotropic elasticity were also performed for comparisons following the model expressions obtained by Choi and Earmme (2002b). In this case, the configuration of a $\mathrm{Ni}$ bi-crystal is considered such that $G_{\mathrm{II}}=\lambda G$ and $k_{\mathrm{II}}=\lambda k$, where $G$ is the shear modulus and $k$ the bulk modulus of $\mathrm{Ni}$, which can be found in Table 2 . Hence, when $\lambda=1$, the configuration reduces to the homogeneous material model and no convergence step is needed $(\beta=0)$. It is found that for a same value of $\lambda$, the convergence is faster in the isotropic elastic case than in the anisotropic one. 


\begin{tabular}{|c|c|c|c|c|c|c|}
\hline & $\lambda$ & 0.25 & 0.5 & 1.0 & 2.0 & 4.0 \\
\hline \multirow{2}{*}{$\boldsymbol{\beta}$} & Anisotropic & 9 & 5 & 3 & 5 & 9 \\
\hline & Isotropic & 7 & 4 & 0 & 4 & 7 \\
\hline
\end{tabular}

Table 3. Number of steps $\beta$ to reach numerical convergence according to the criteria $\left(\epsilon_{F}^{\beta+1}\right.$,

$\epsilon_{u}^{\beta+1}$ and $\epsilon_{\sigma}^{\beta+1}<10^{-4}$ ) for different values of $\lambda$ : anisotropic vs. isotropic elastic cases.

\subsection{Effects of grain boundary modelling characteristics}

This sub-section considers a single straight edge dislocation in a Ni bi-crystal and compares the induced elastic fields for different kinds of grain boundary (GB) modelling characteristics: zero thickness GB (bi-material configuration) or GB with $H=5|\boldsymbol{b}|$ and different values of the stiffness parameter $\lambda$ (tri-material configuration). It must be underlined that such description, of a GB is a simplified one as it ignores its internal structure and composition (e.g., in terms of GB defects or interfacial elasticity considering intrinsic GB elastic energy). The interface or the middle of the GB is fixed at $x_{2}=0$ and the distance from the interface or the middle of the GB to the dislocation along the slip direction is $L(1)=10|\boldsymbol{b}|$. The other parameters are set to the values presented in Table 2.

Figures $8 \mathrm{a}$ and $8 \mathrm{~b}$ show contour plots of the displacement component $u_{1}$ and stress components $\sigma_{12}$ which are induced by a single edge dislocation in a Ni bi-crystal with a zero thickness GB and with GBs characterized by $H=5|\boldsymbol{b}|$ and $\lambda=0.5,1$ and 2 (tri-material model), as well as the differences between bi-material model and tri-material models. It is remarked that $u_{1}$ and $\sigma_{12}$ are continuous across interfaces, which is consistent with continuity conditions. In the case of $\lambda=1$, for both fields, the greatest differences are located in the middle of the GB. In the case $\lambda=0.5$ and 2 , the differences are mainly located around the first (or upper) interface and are also much larger compared to the case $\lambda=1$. The cases $\lambda=0.5$ (compliant GB) and $\lambda=2$ (stiff GB) often display opposite effects. For example, the difference of displacement $u_{1}$ is negative below the dislocation when $\lambda=0.5$ while it is positive when $\lambda=2$. 

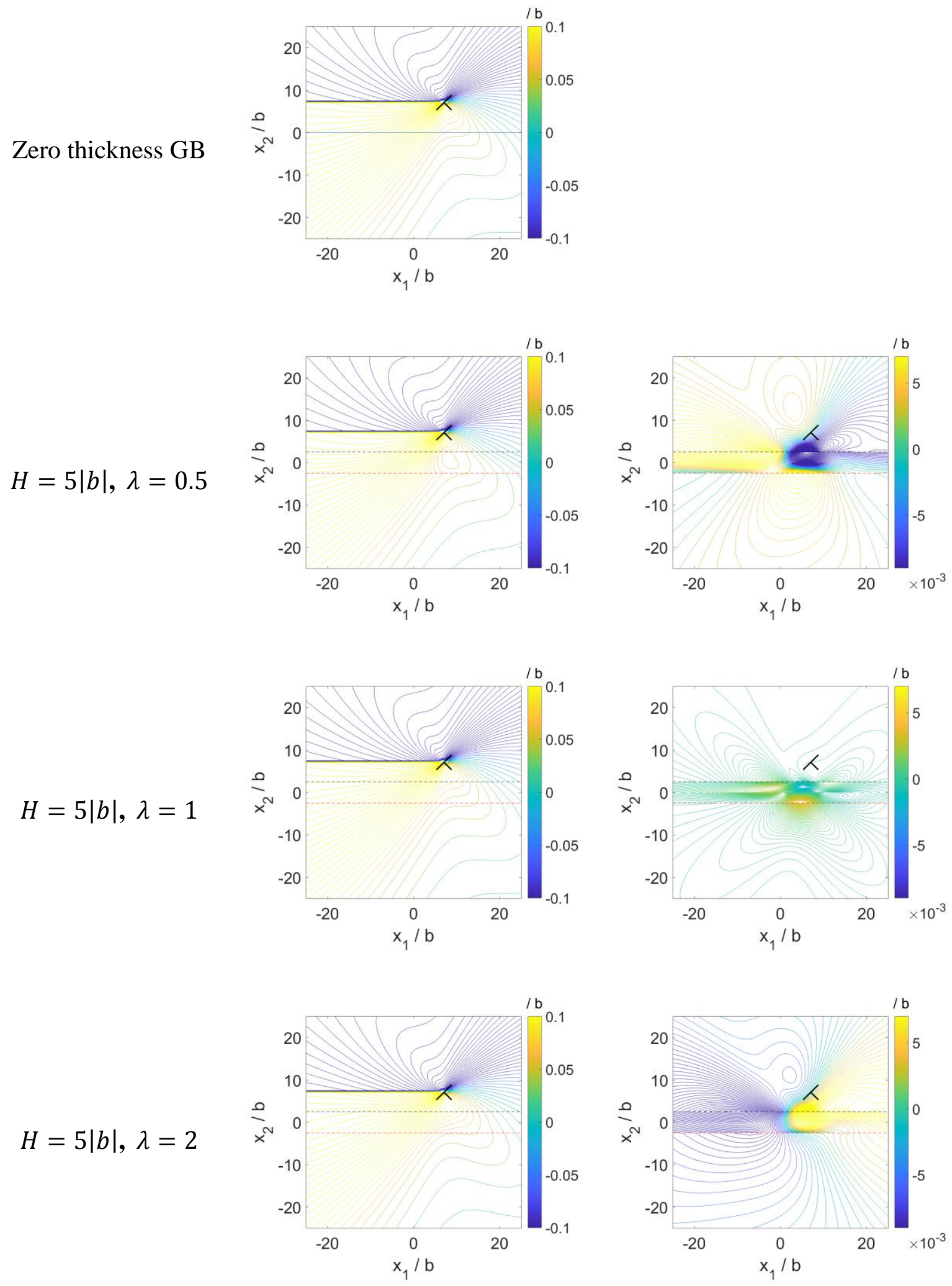

Figure 8 (a). Displacement $\mathrm{u}_{1}$ induced by a single dislocation in a Ni bi-crystal with different GB models (left column), as well as the differences with respect to the zero thickness GB case (right column). 

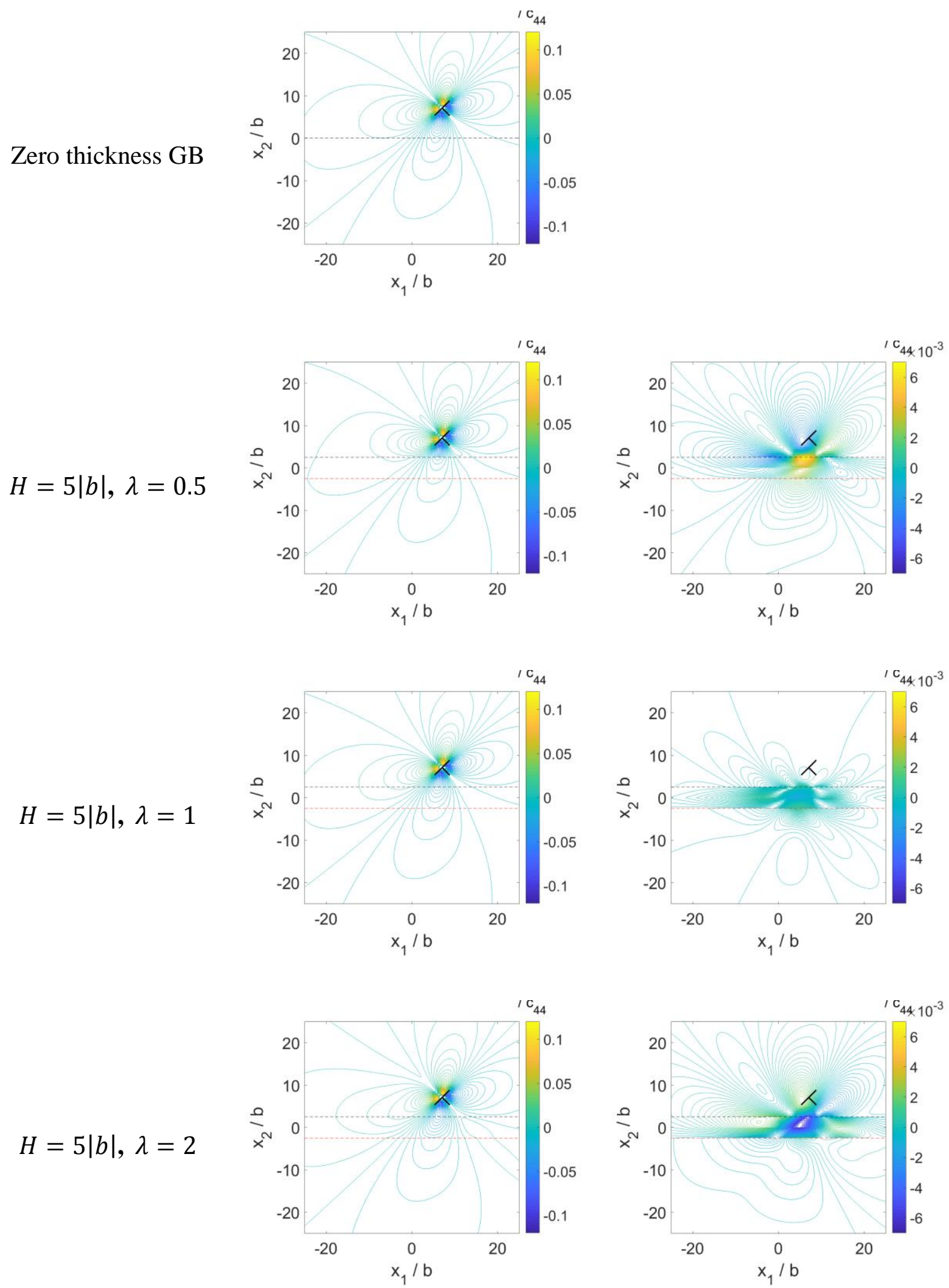

Figure 8 (b). Stress $\sigma_{12}$ induced by a single dislocation in a Ni bi-crystal with different GB models (left column), as well as the differences with respect to the zero thickness GB case (right column). 


\subsection{Analysis of image force effects}

This sub-section considers a single straight edge dislocation in a $\mathrm{Ni}$ bi-crystal or in a $\mathrm{Ni}$ half-space. In such heterogeneous medium, an image force is exerted on the dislocation due to the presence of interface(s) or surface. In the absence of applied stress, the projection of the P-K force along $\boldsymbol{v}$ reduces to (Hirth and Lothe, 1982):

$$
F_{i m}=\left[\left(\sigma_{i n t} \cdot \boldsymbol{b}\right) \times \boldsymbol{t}\right] \cdot \boldsymbol{v}
$$

As mentioned in the previous sub-sections, it is always considered that $\boldsymbol{v}$ points towards the GB or the surface. Hence, $F_{i m}>0$ means that the dislocation is attracted by the GB or the surface whereas $F_{i m}<0$ means that the dislocation is repelled by the GB or the surface.

Hereafter, the misorientation effect between both crystals is investigated. Both orientations $\mathrm{A}^{\text {Ori }}$ and $\mathrm{B}^{\mathrm{Ori}}$ are considered for the lower crystal while the other parameters are set to the default values reported in Table 2. The results are presented in Figure 9 and Figure 10. In the legends of the figures, “Ani”, "Iso", "rigid Half-space”, "free Half-space”, "bi”, “A Ori”, and "B Ori”, indicate elastic anisotropy, elastic isotropy, half space with rigid surface, half space with free surface, bi-material model, orientation $\mathrm{A}^{\text {Ori }}$ for lower crystal and orientation $\mathrm{B}^{\text {Ori }}$ for lower crystal, respectively.

First, Figure 9 (a) considers half-space configurations and Figure 9 (b) considers bi-materials (i.e., zero thickness GB). As expected, it indicates that in a half space, a rigid surface has always a repulsive effect on the dislocation. On the contrary, a free surface has always an attractive effect. Moreover, if the lower crystal is set to orientation $\mathrm{A}^{\text {Ori }}$, the projected image force $F_{i m}$ is always positive, which means that the zero thickness GB exerts an attractive effect on the dislocation, whereas with orientation $\mathrm{B}^{\text {Ori }}$, the dislocation is repelled by the GB. It can be also noticed that the magnitude of $F_{i m}$ is far smaller for the bi-crystals than for the half-spaces with free / rigid surfaces, which represent two extreme cases. Besides, considering isotropic elasticity in half-spaces lead to slightly larger magnitude of $F_{i m}$ than considering anisotropic elasticity but the differences remain very small. Given the chosen orientation of upper crystal, it should be pointed out that orientation $\mathrm{A}^{\text {Ori }}$ and orientation $\mathrm{B}^{\text {Ori }}$ of lower crystal produce the maximum positive and negative projected image forces $F_{i m}$, respectively. 
(a)

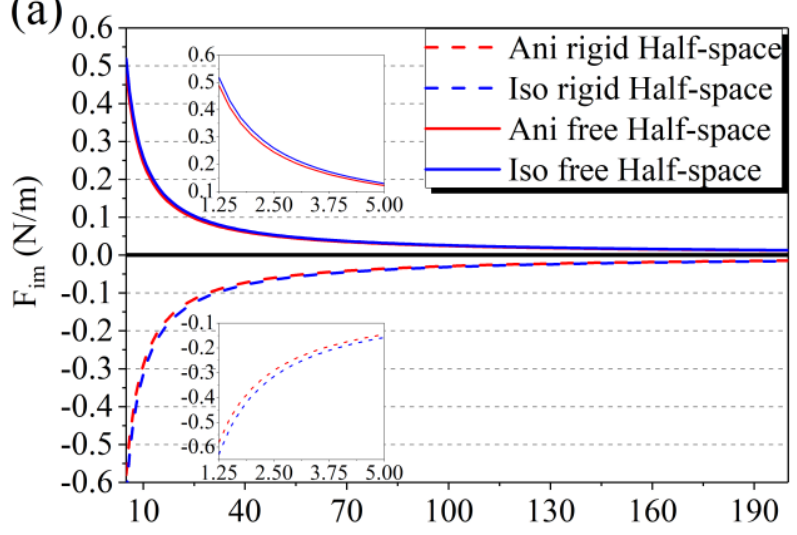

Distance from surface along the slip direction $(|b|)$

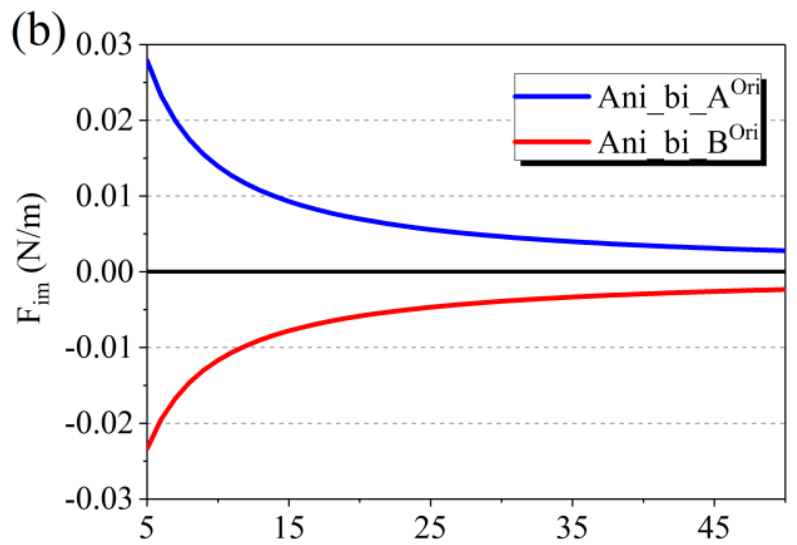

Distance from GB along the slip direction $(|b|)$

Figure 9. Variation of the projected image force $F_{\text {im }}$ with the distance from the GB along the slip direction for different configurations: half space with anisotropic vs. isotropic elasticity (a) and bi-crystal modeled by bi-material configurations (b).

Figure 10 considers only tri-material configurations with $H=5|\boldsymbol{b}|$. Considering isotropic elasticity, the projected image force $F_{i m}$ is always negative in case of a stiff GB $(\lambda=2)$ and positive in case of a soft GB $(\lambda=0.5)$. Interestingly, in the case of anisotropic elasticity, $F_{i m}$ may exhibit a change of sign and extremal points because of the coupled effects of the image forces arising both from the lower crystal and the GB. For instance, considering a GB characterized by $\lambda=2$ and orientation $\mathrm{A}^{\text {Ori }}$ for the lower crystal, $F_{i m}$ is negative close to the GB and becomes positive beyond $41|\boldsymbol{b}|$ from the center of GB (Figure 10 (a)). The repelling effect close to GB is due to the larger stiffness of the GB $(\lambda=2)$ while the attractive effect that becomes predominant at long distance is due to orientation $\mathrm{A}^{\text {Ori }}$ of the lower crystal as shown in Figure 9 (b). Accordingly, an equilibrium position for the dislocation can be defined where $F_{i m}=0$. In a similar manner, an equilibrium position in the absence of applied stress can also be found by coupling an attractive compliant GB $(\lambda=0.5)$ with the repulsive orientation $\mathrm{B}^{\mathrm{Ori}}$ of the lower crystal (Figure 10). In this case, the equilibrium position of the dislocation is found around $58|\boldsymbol{b}|$ along the slip direction from the center of GB. It is noteworthy that the image forces of Figure 10 are one order of magnitude lower than the ones reported for anisotropic bi-materials (Figure 9 (b)). 


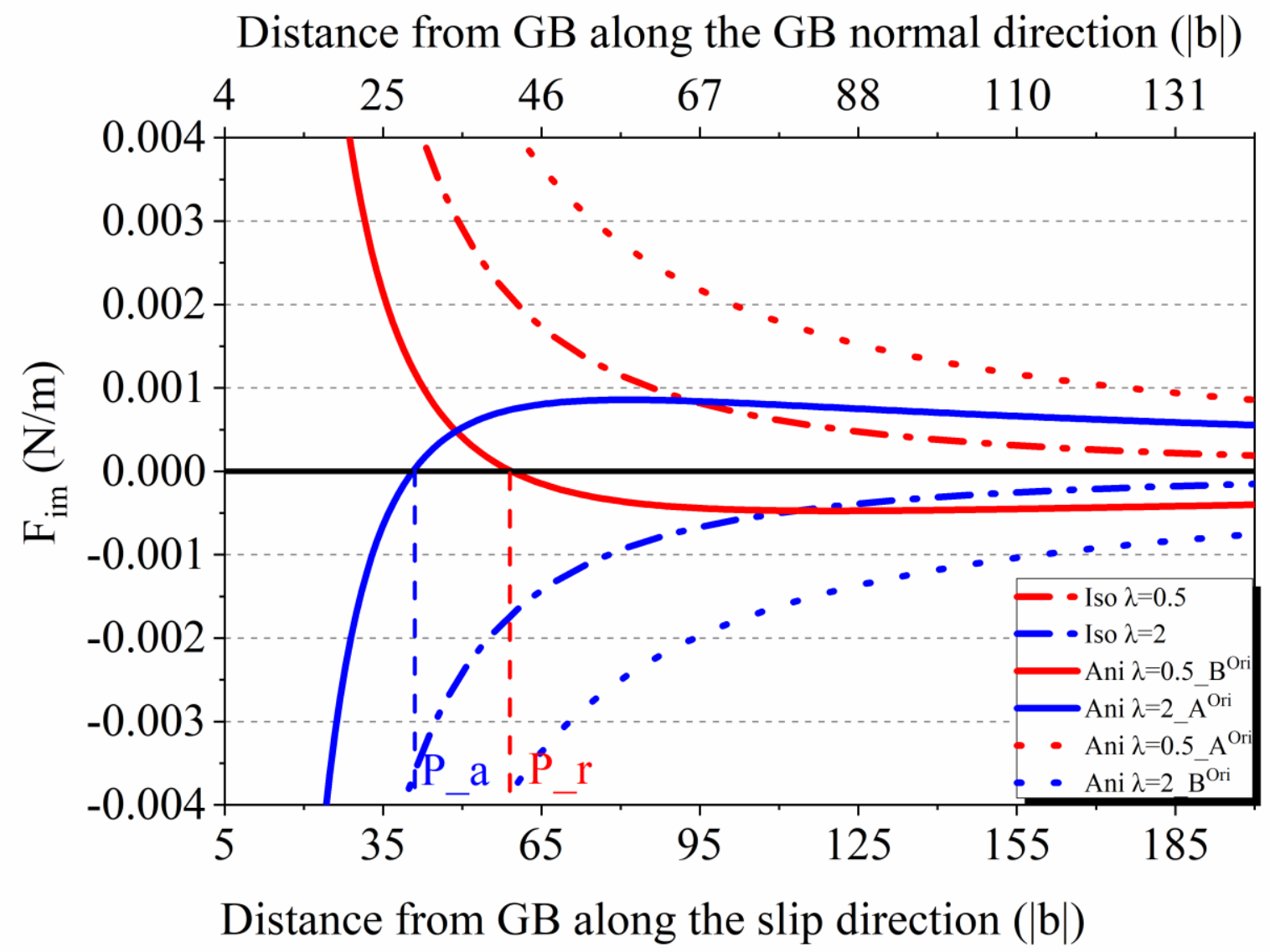

Figure 10. Variation of the projected image force $\mathrm{F}_{\mathrm{im}}$ with the distance from GB for different tri-material configurations with $\mathrm{H}=5|\mathbf{b}|$. Anisotropic vs. isotropic elasticity.

\subsection{Results and discussion for dislocation pile-ups with locked leading dislocation}

As mentioned in section 7.1, for dislocation pile-up configuration, the position of the first (or leading) dislocation is locked at $L(1)=5|\boldsymbol{b}|$ and the other parameters are set to the default values presented in Table 2. Comparisons are made for different GB modelling characteristics, as well as between pile-ups having different total number $N$ of dislocations. As a result, the position of each dislocation in the pile-up, the displacement and stress fields are obtained.

\section{$\underline{\text { 7.5.1. Dislocation pile-up lengths }}$}

The results for dislocation pile-up length for different numbers of dislocations are summarized in Table 4. From Table 4, it can be inferred that the dislocation pile-up length increases with the GB stiffness (from $\lambda=0.5$ to $\lambda=2$ ), whatever the total number of 
dislocations considered in the pile-up. This effect is, however, very weak with a variation of less than $1 \%$. As discussed in section 6.1, the P-K force is not zero on the leading dislocation. The force on the locked dislocation due to the other dislocations and the applied stress may indeed exceed the image force arising from the GB. Moreover, it is observed that the pile-up length obtained with $\lambda=2$ and orientation $\mathrm{A}^{\text {Ori }}$ is smaller than the one obtained for the homogenous anisotropic crystal (Table 4). As discussed in section 7.4, if the GB with $\lambda=2$ exerts a repelling effect, orientation $\mathrm{A}^{\text {Ori }}$ of the lower crystal exerts an attractive effect so that the global effect on dislocations beyond the equilibrium point is attractive. It is noteworthy that the equilibrium distance is about $10.25 \mathrm{~nm}$, which represents a short distance compared to the pile-up length. This means that nearly all the dislocations undergo attractive effects (Figure 10). By comparison, in a homogeneous single crystal, there is neither attractive nor repulsive effect due to boundaries and thus the dislocation pile-up length is larger. A similar analysis explains why the dislocation pile-up length obtained with $\lambda=0.5$ and orientation $\mathrm{B}^{\mathrm{Ori}}$ is larger than the one obtained for the homogenous anisotropic crystal since orientation $\mathrm{B}^{\text {Ori }}$ exerts a global repulsive effect on the dislocations of the upper crystal. Table 4 indicates clearly that going from an attractive to a repulsive orientation for the lower crystal has much more impact on the pile-up length (variation of about $8 \%$ ) than changing $\lambda$ from 0.5 to 2 (variation less than 1\%).

In the isotropic case, the dislocation pile-ups length is always larger when considering a GB with $\lambda=2$, and smaller if $\lambda=0.5$, compared to the homogeneous single crystal case since there is no misorientation effect. A strong effect on the pile-up length is only obtained when considering a half-space with a rigid surface (variation of 100\%) since such interface displays the strongest repulsive image force on dislocations that one can obtain.

As mentioned in section 6.2, for a large number of dislocations in isotropic elasticity, the pile-up length can be approximated by $L=\frac{2 N A}{\tau_{0}}$ with $A=\frac{G|\boldsymbol{b}|}{2 \pi(1-v)}$ for edge dislocations (Eshelby et al., 1951). For $N=50$ and the other parameters are set to the default values presented in Table 2, the approximated pile-up length for $\mathrm{Ni}$ is $10.375 \mu \mathrm{m}$. This length is 
11.9\%. greater than the value presented in Table 4 for isotropic homogeneous crystal $L=9.274 \mu m$. 
Table 4. Pile-up length L for different configurations considering anisotropic vs. isotropic elasticity: homogeneous crystal, bi-material, tri-material with GB stiffness characterized by $\lambda$, rigid half-space.

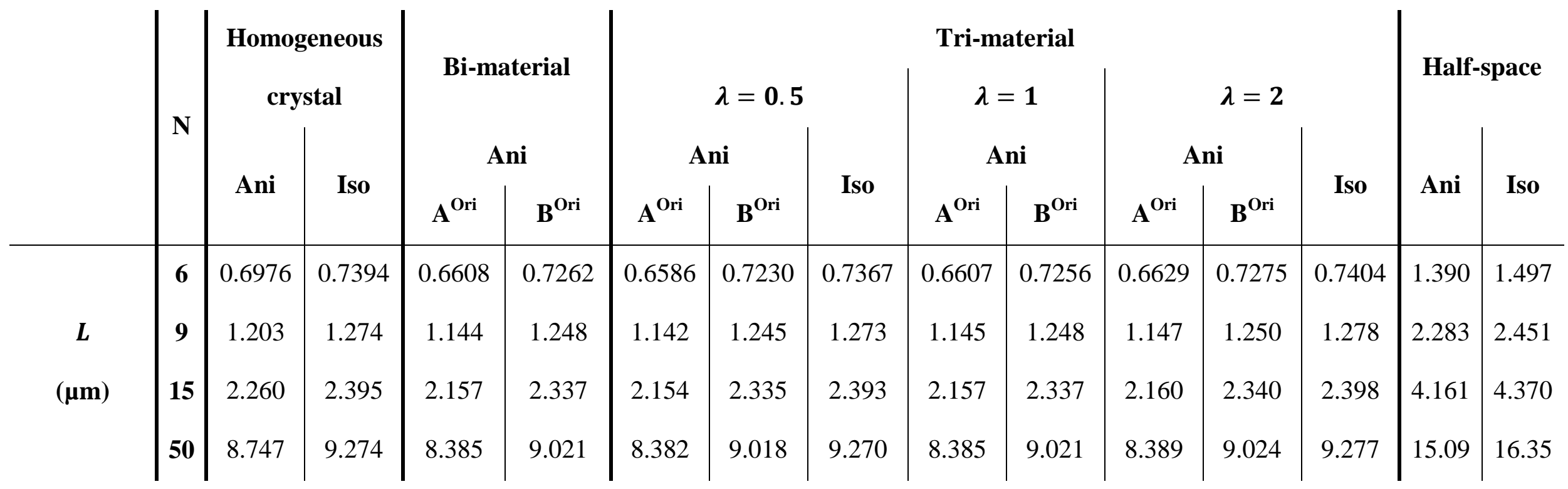


Since the orientation of the lower crystal has a strong effect on the dislocation pile-up length $L$ for anisotropic elasticity, it is interesting to see the variation of $L$ with a continuous variation of this orientation. Figure 11 shows such variation of $L$ in case of a pile-up with 50 dislocations and a zero thickness GB. The orientation of the upper crystal (where there is the pile-up) is fixed, still defined by $\alpha=45^{\circ}$, whereas the orientation of lower crystal is given by a rotation $\psi$ around $\boldsymbol{v}$. In Figure 11, the rotation angle $\psi$ varies continuously from $0^{\circ}$ to $180^{\circ}$. Hence, the homogenous single crystal case is retrieved for $\psi=0^{\circ}$ and $\psi=180^{\circ}$ for which $L=8.75 \mu \mathrm{m}$ (Figure 11). As a consequence, when $L$ is smaller (resp. larger) than $8.75 \mu \mathrm{m}$, it can be inferred that the image force has globally an attractive (resp. repulsive) effect on the pile-up. Figure 11 also displays the misorientation angle between both crystals, i.e. the minimum rotation angle to get from one crystal orientation to the other. In the present example, the maximal possible value for misorientation angle in cubic crystal, $62.8^{\circ}$ (Mackenzie and Thomson, 1957), is almost reached but does not correspond to an extremal value of $L$. The maximal value of $L$ corresponds however to a high misorientation angle (about $60^{\circ}$ ). Furthermore, it is noteworthy that a same misorientation angle can be related to different elastic behaviors corresponding to different pile-up lengths (for example, $\Phi=60^{\circ}$ is retrieved with $\psi=60^{\circ}$ and $\psi=120^{\circ}$, which give $L=9.02 \mu \mathrm{m}$ and $L=8.61 \mu \mathrm{m}$, respectively, see Figure 11). Thus, besides the misorientation angle, attention should also be paid on misorientation axis as many different mechanical behaviors can be associated to a same misorientation angle. Finally, it can be observed that the relative variations of $L$ in $\mathrm{Ni}$ can reach about $7 \%$. The important point is that such significant variations occur in a moderately elastic anisotropic material and cannot be captured considering pile-ups in isotropic elasticity. Furthermore, the average of $L$ for anisotropic elasticity is about $L=8.76 \mu \mathrm{m}$. And compared with anisotropic half space $L=15.09 \mu \mathrm{m}$ (Table 4) and isotropic half space $L=16.35 \mu \mathrm{m}$ (Table 4), the average length is only $58.1 \%$ and $53.6 \%$ of the pile-up length for anisotropic and isotropic half space, respectively. 


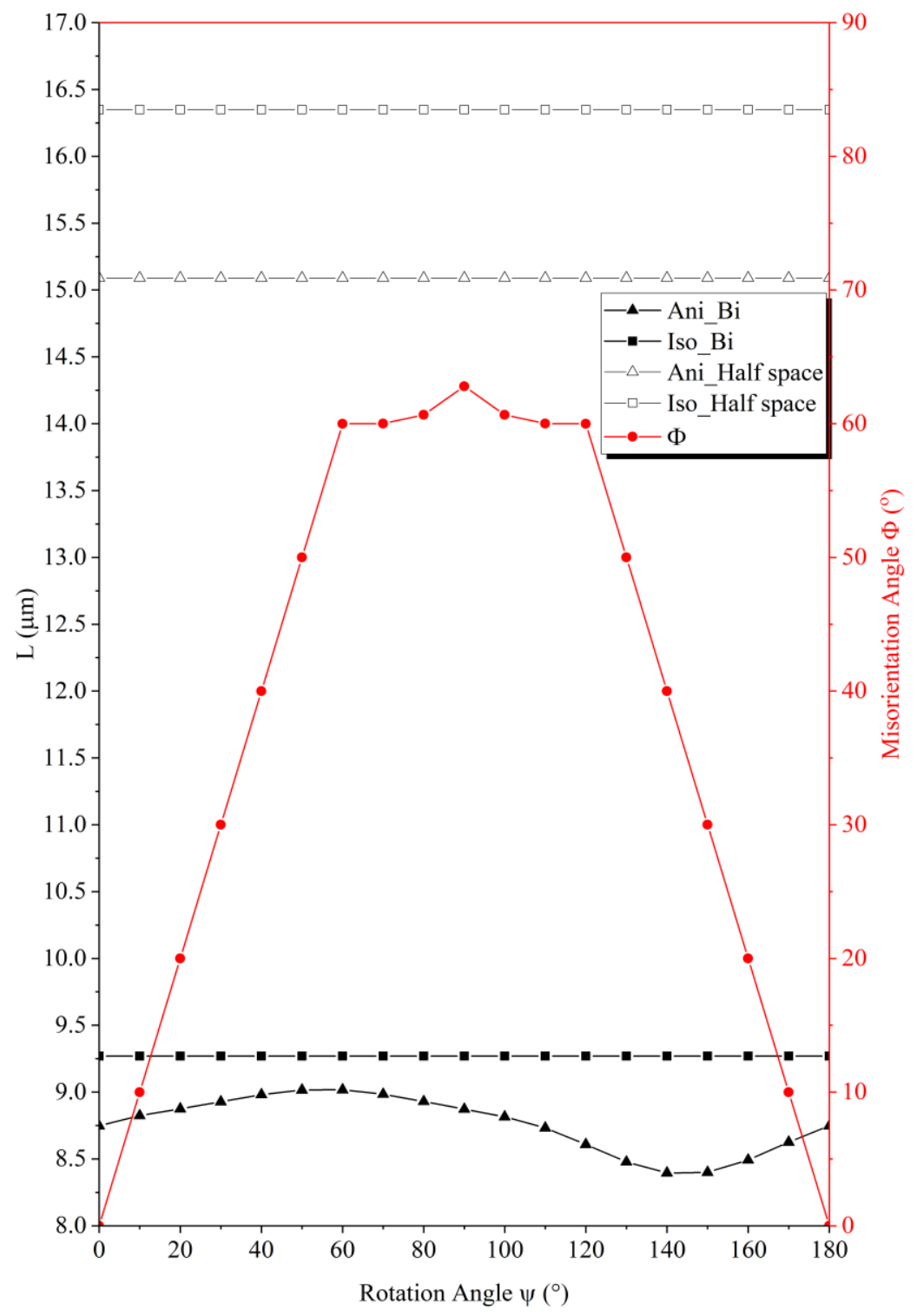

Figure 11. Misorientation effect on pile-up length L for a pile-up with 50 dislocations and an anisotropic bi-crystal with zero thickness GB (Ani_Bi). The rotation axis is the glide direction v. Comparisons with the homogeneous isotropic case (Iso_Bi), the isotropic half-space (Iso_Half space) and the anisotropic half-space (Ani_Half space).

\subsubsection{Resolved shear stress profiles}

From Eq. ( 29 ) of section 6.2, the lower bound estimate of the resolved shear stress in front of a dislocation pile-up can be written in isotropic homogeneous elasticity as: 


$$
\frac{\tau_{\text {int }}+\tau_{0}}{\tau_{0}}=\frac{1}{\sqrt{\frac{d}{L}}} \quad \text { for } \frac{1}{15} l \ll d \ll L
$$

where $\tau_{\text {int }}$ is the resolved shear stress produced by all the dislocations of the pile-up. Figure 12 shows then the variation of $\frac{\tau_{i n t}+\tau_{0}}{\tau_{0}}$ with respect to $\frac{d}{L}$ for 50 edge dislocations and different kind of configurations (in case of heterogeneous medium, $\tau_{\text {int }}$ includes the effects of image forces). In order to compare the present results with the work of Eshelby et al. (1951) in isotropic elasticity, the value of $L$ used for normalization always corresponds to the approximation $\frac{2 N A}{\tau_{0}}$.

From Figure 12 (a), it can be seen that the approximation of a resolved shear stress decrease with the inverse of the square root distance from the pile-up remains valid in anisotropic elasticity and for a heterogeneous medium. All the plots are relatively close to each other. The results display also some influence of the second crystal orientation in case of a bi-material model. The resolved shear stress with orientation $\mathrm{A}^{\text {Ori }}$, which exerts an attractive effect on dislocations, is smaller than the one obtained with orientation $\mathrm{B}^{\text {Ori }}$, which exerts a repulsive effect on dislocations. Thus, in the studied case, the repulsive orientation leads to slightly higher resolved shear stress in front of the dislocation pile-up compared to the attractive orientation.

Figure 12 (b) represents the results obtained with the tri-material model. It is found that, with the same orientation of the second crystal, the resolved shear stress are nearly the same in the area of $\frac{1}{15} l \ll d \ll L$ for $\lambda=0.5$ and $\lambda=2$. The reason is that the thickness of the interphase is very small $(\mathrm{H}=5|\mathbf{b}|)$, and its stiffness has not been varied too much. Hence, the effect of interphase stiffness is negligible compared to the one of misorientation. Similar misorientation effects as obtained with the bi-material model are indeed retrieved: the attractive orientation $\mathrm{A}^{\text {Ori }}$ gives lower resolved shear stress than the repulsive orientation $\mathrm{B}^{\text {Ori }}$. 

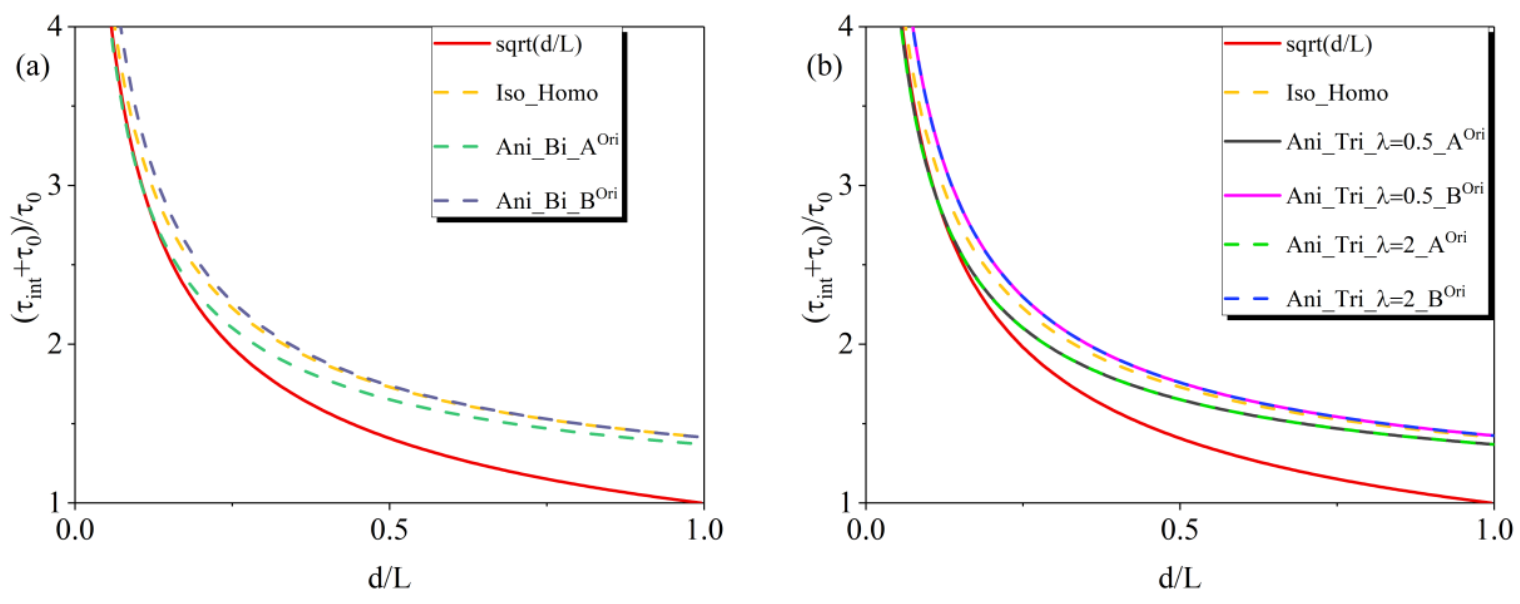

Figure 12. Eshelby et al's diagrams: resolved shear stress along the slip direction in front of a dislocation pile-up with 50 edge dislocations for different configurations.

\subsection{Results and discussion for dislocation pile-ups without locked leading dislocation}

As mentioned in section 6.1, in an heterogeneous medium, the boundary image force on the leading dislocation may balance the applied stress and the stress contribution coming from other dislocations (Wagoner, 1981), but only in case of repulsive image force. So in this case, it is not needed to lock the leading dislocation in the pile-up. All the dislocation positions, including the leading one, can be found by the iterative relaxation scheme. From the results discussed in section 7.4 , for a tri-material model with a small thickness $\mathrm{H}=5|\mathbf{b}|$, the image force is mainly dependent on the orientation of neighboring crystal at long distance from GB. In the region close to GB, a coupled effect due to both the neighboring crystal's orientation and GB stiffness occurs. To investigate the influence of GB stiffness on real dislocation positions, the orientation of neighboring crystal is chosen as orientation $\mathrm{B}^{\text {ori }}$, which gives a maximal repelling effect. In addition, the GB stiffness varies from $\lambda=0.925$ to $\lambda=2$, which could reflect a compliant or a stiff GB. The minimum value of $\lambda$ is chosen as 0.925 in order to lead to a stable dislocation pile-up. If $\lambda$ is too small, the positive image force close to GB will be very large (attractive effect) and therefore the dislocation pile-up would not be equilibrated. To study a difference between different GB stiffnesses, the applied stress field is chosen lower than in section 7.5 (see Table 2), with $\sigma_{22}^{\text {ext }}=50 \mathrm{MPa}$. The other parameters are set to the default values presented in Table 2. Comparisons are made for different GB 
stiffnesses $\lambda$ and different total number $\mathrm{N}$ of dislocations within the pile-up. As a result, the equilibrium position of each dislocation in the pile-up and the resolved shear stress in the neighboring grain are obtained.

\subsubsection{Position of dislocations in the pile-up and dislocation pile-up length}

As the leading dislocation is no longer locked, its position is modified when different GB stiffnesses are considered as well as the positions of the other dislocations and the pile-up length. The results are presented in Figure 13 where $L(N)-L(1)$ defines the pile-up length. For a fixed number of dislocations in the pile-up $(N=5,10$ and 20$)$, the equilibrium position of each dislocation inside the pile-up is more and more far away from the GB as $\lambda$ is larger as shown in Figure 13. Besides, the pile-up length $L$ almost linearly scales with $\lambda$ following the red lines displayed in Figure 13 (a)-(c). It is noteworthy that Lubarda (2017) also found an increase of pile-up length $L$ in the case of stiffer inhomogeneity for isotropic bi-materials. Here, a similar effect is obtained in the context of heterogeneous anisotropy. When the number of dislocations in the pile-up increases from $N=5$ to $N=20$ and for a same $\lambda$, the pile-up length also increases following Figure 13 (a)-(c). For $N=5$, the pile-up length increases with a magnitude of $1.5 \mathrm{~nm}$ when $\lambda$ varies from 0.925 to 2 . For $N=10$ and $\mathrm{N}=20$, the pile length increases with magnitudes $2.0 \mathrm{~nm}$ and $2.5 \mathrm{~nm}$, respectively. As a conclusion, the more dislocations there are in the pile-up, the larger the increase of pile-up length with $\lambda$. 


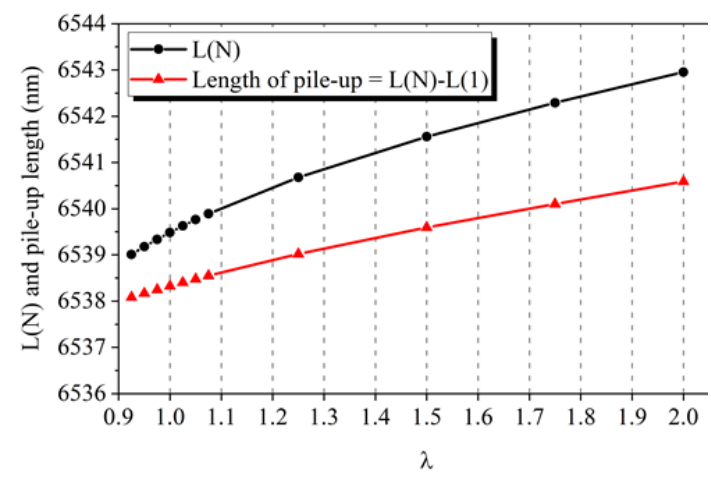

Figure 13 (a)-(c) Dislocation pile-up length and position of last dislocation in the pile-up for $\mathrm{N}=5,10$ and 20, respectively. (d) First (not fixed) leading dislocation position in the pile-up for $\mathrm{N}=5,10$ and 20 with different GB stiffnesses characterized by $\lambda=0.925$ to $\lambda=2$.

\subsubsection{Resolved shear stress profiles}

As discussed in section 7.5 when the leading dislocation is locked, the resolved shear stress along the slip direction is nearly the same for the same orientation of lower crystal with different $\lambda$ in the area of $\frac{1}{15} l \ll d \ll L$. For the unlocked case, the values of resolved shear stresses at a distance of $|\mathbf{b}|$ from the second interface along the slip direction for $\lambda$ varying from 0.925 to 2 and different $N$ are shown in Figure 14. It is seen that the resolved shear stress always decreases when $\lambda$ increases for different number of dislocations in pile-up due to the pushing back of dislocations from GB. For $N=5,10$ and 20, the resolved shear stress decrease from $\lambda=0.925$ to $\lambda=2$ is $1446.3 \mathrm{MPa}, 1301.6 \mathrm{MPa}$ and 1058.4 $\mathrm{MPa}$, respectively. When $\mathrm{N}$ is large $(\mathrm{N}=20)$, Figure 14 shows a slower decrease of the resolved shear stress with $\lambda$. From Figure 14, it is shown that for $\lambda=0.9 \sim 1.1$, the resolved shear stress decreases faster than for $\lambda>1.1$. The influence of $N$ is more important for a GB stiffness from $\lambda=0.9$ to $\lambda=1.1$ than for $\lambda>1.1$. 


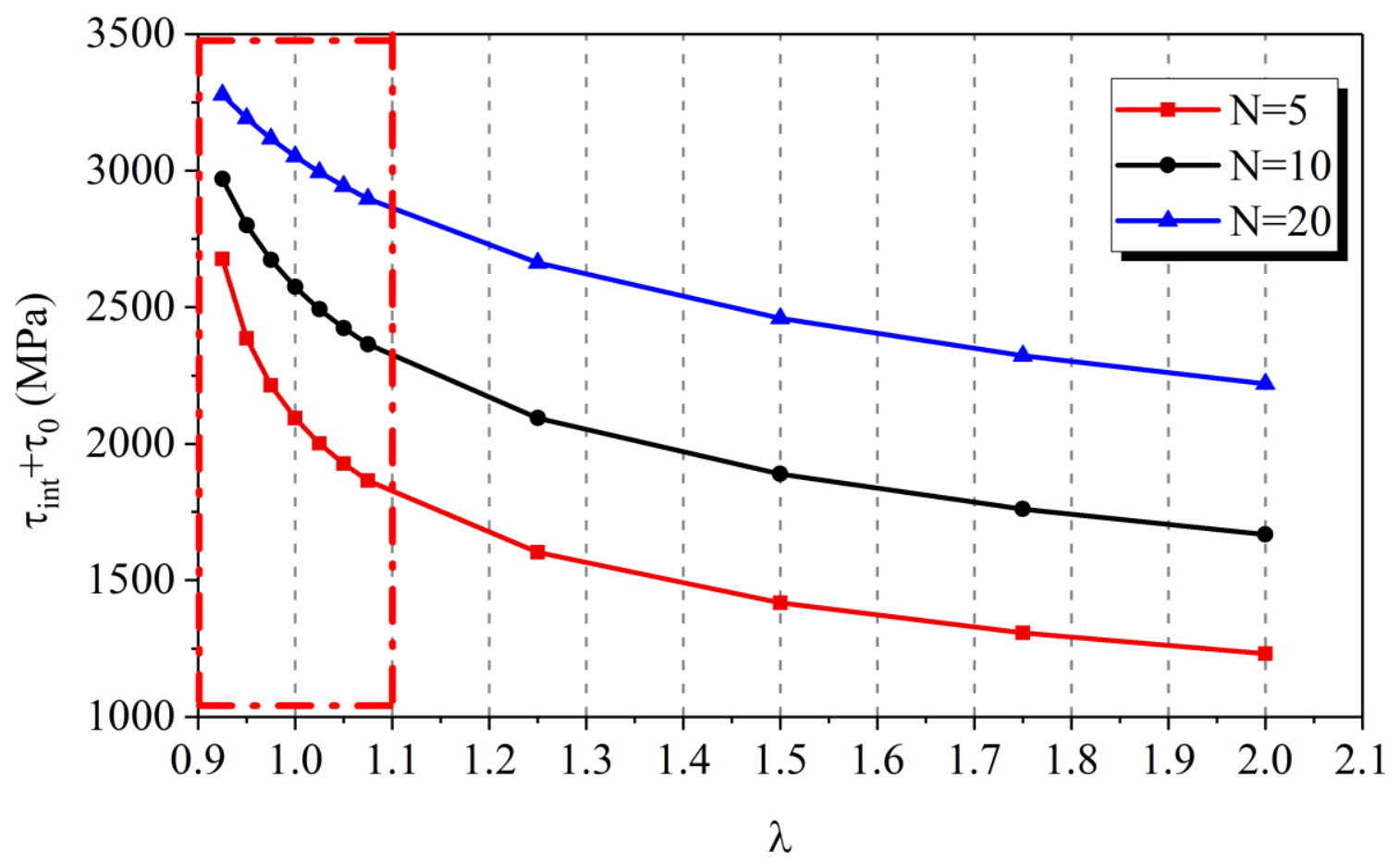

Figure 14. Resolved shear stresses in the neighboring grain at a distance of $|\mathbf{b}|$ from the second interface along the slip direction.

\section{Conclusions}

The present paper considered the Leknitskii-Eshelby-Stroh (LES) formalism for two-dimensional anisotropic elasticity to compute elastic fields due to single dislocations and dislocation pile-ups in heterogeneous media like bi-materials, half-spaces and tri-materials. For the latter, the solutions were derived using the alternating technique developed by Choi and Earmme (2002a). The tri-material configuration was used to consider grain boundary (GB) regions with non-zero thickness and specific GB stiffness thanks to the parameter $\lambda$.

After studying the convergence of the series solutions for tri-material configuration with two new criteria based on stress and displacement fields in the whole simulation box, the effects of GB modelling characteristics were considered. It was first found that the Peach-Koehler (P-K) force on a single edge dislocation in a Ni bi-crystal with different GB stiffness can exhibit a change of sign with the dislocation position. In this configuration, an equilibrium position in the absence of applied stress was found as the result of the competition between an attractive 
compliant GB with a repelling neighboring grain, or a repelling stiff GB with an attractive neighboring grain.

For discrete edge dislocation pile-ups in bi-crystals with different GB stiffness and different misorientations with or without leading locked dislocation, the different conclusions are sketched below:

- The pile-up length for different configurations considering anisotropic versus isotropic elasticity has been computed for different situations: homogeneous crystal, bi-material, tri-material with GB stiffness characterized by $\lambda$ from 0.5 to 2 , rigid half-space. From these configurations and following the numerical results, it was concluded that the first effect on the dislocation pile-up length is the crystallographic misorientation between grains compared to the intrinsic GB stiffness. For a given misorientation, the pile-up length is always much lower than the one resulting from anisotropic or isotropic rigid half-space.

- From the calculations of resolved shear stress profiles in the neighboring grain, it was shown using Eshelby et al's diagram (see Figure 12) that the approximation of a shear stress decrease with the inverse of the square root distance from the pile-up remains valid in the case of anisotropic and heterogeneous elasticity.

- In the case of stiffer GB (unlocked pile-up configurations), an increase of the dislocation pile-up length was observed to be more important as the number of dislocations in the pile-up and the GB stiffness are higher. The resolved shear stress at short distance of the GB in the neighboring grain exhibits a decrease in magnitude as the GB stiffness increases. If the parameter $\lambda$ is low, then a stronger decrease was observed.

The results of this paper may be used to improve the theoretical modeling of GB elastic stiffness in Discrete Dislocation Dynamics (DDD) numerical codes including anisotropic elasticity, since the often used assumption of impenetrable rigid GB repelling dislocations may lead to unrealistic pile-up lengths in polycrystalline materials.

As future work, it is intended to consider dislocation pile-ups with more compliant GBs and absorbed dislocations in the GB region to study slip transfer configurations in comparison with experimental results on $\mathrm{Ni}$ bi-crystals. 


\section{Acknowledgements}

The authors are grateful to the French Ministry of Higher Education and Scientific Research and the French-German University (UFA-DFH) for financial supports.

\section{References}

Alshits, V.I., Kirchner, H.O.K., 1995. Elasticity of multilayers I. Basic equations and solutions. Philosophical Magazine A 72, 1431-1444. https://doi.org/10.1080/01418619508243922

Asaro, R.J., Lubarda, V.A., 2006. Mechanics of solids and materials. Cambridge University Press, Cambridge.

Bacon, D.J., Barnett, D.M., Scattergood, R.O., 1979. Anisotropic continuum theory of lattice defects. Progress in Materials Science 23, 51-262. https://doi.org/10.1016/0079-6425(80)90007-9

Barnett, D.M., 1967. The effect of shear modulus on the stress distribution produced by a planar array of screw dislocations near a bi-metallic interface. Acta Metallurgica 15, 589-594. https://doi.org/10.1016/0001-6160(67)90101-0

Barnett, D.M., Lothe, J., 1973. Synthetis of the sextic and the integral formalism for dislocations, Green's functions and surface waves in anisotropic elastic solids. Physica Norvegica 7, 13-19.

Barnett, D.M., Lothe, J., 1974. An image force theorem for dislocations in anisotropic bicrystals. J. Phys. F: Met. Phys. 4, 1618. https://doi.org/10.1088/0305-4608/4/10/010

Bonnet, R., Dupeux, M., 1980. Stress calculations for interfacial extrinsic and intrinsic dislocation arrays in anisotropic two-phase media. Philosophical Magazine A 42, 809-812. https://doi.org/10.1080/01418618008239386

Chadwick, P., Smith, G.D., 1977. Foundations of the Theory of Surface Waves in Anisotropic Elastic Materials, in: Yih, C.-S. (Ed.), Advances in Applied Mechanics. Elsevier, pp. 303-376. https://doi.org/10.1016/S0065-2156(08)70223-0

Choi, S.T., Earmme, Y.Y., 2002a. Elastic study on singularities interacting with interfaces using alternating technique. International Journal of Solids and Structures 39, 943-957. https://doi.org/10.1016/S0020-7683(01)00230-X

Choi, S.T., Earmme, Y.Y., 2002b. Elastic study on singularities interacting with interfaces using alternating technique. International Journal of Solids and Structures 39, 1199-1211. https://doi.org/10.1016/S0020-7683(01)00231-1

Chou, Y.T., 1966. Equilibrium of Linear Dislocation Arrays in Heterogeneous Materials. Journal of Applied Physics 37, 2425-2429. https://doi.org/10.1063/1.1708830

Chou, Y.T., Li, J.C.M., 1969. Theory of Dislocation Pileups, in: "Proceedings of the Symposium on the Mathematical Theory of Dislocations", ASME Publication, pp.116-177. 
Chou, Y.T., Pande, C.S.,Yang, H.C. 1975. Interfacial edge dislocations and dislocation walls in anisotropic two-phase media. Journal of Applied Physics 46, 5-10. http://dx.doi.org/10.1063/1.321369

Chu, H., Pan, E., Han, X., Wang, J., Beyerlein, I.J., 2012. Elastic fields of dislocation loops in three-dimensional anisotropic bimaterials. Journal of the Mechanics and Physics of Solids 60, 418-431. doi:10.1016/j.jmps.2011.12.007

Chu, H., Pan, E., 2014. Elastic fields due to dislocation arrays in anisotropic bimaterials. International Journal of Solids and Structures 51, 1954-1961. https://doi.org/10.1016/j.ijsolstr.2014.02.001

Dillon, S.J., Tang, M., Carter, W.C., Harmer, M.P., 2007. Complexion: A new concept for kinetic engineering in materials science. Acta Materialia 55, 6208-6218. doi:10.1016/j.actamat.2007.07.029

Eshelby, J.D., 1951. The Force on an Elastic Singularity. Philosophical Transactions of the Royal Society of London A: Mathematical, Physical and Engineering Sciences 244, 87-112. https://doi.org/10.1098/rsta.1951.0016

Eshelby, J.D., Frank, F.C., Nabarro, F.R.N., 1951. The equilibrium of linear arrays of dislocations. Philosophical Magazine Series 42, 351-364. https://doi.org/10.1080/14786445108561060

Eshelby, J.D., Read, W.T., Shockley, W., 1953. Anisotropic elasticity with applications to dislocation theory. Acta Metallurgica 1, 251-259. https://doi.org/10.1016/0001-6160(53)90099-6

Gemperlová, J., 1968. Force on Dislocations in Anisotropic Bicrystals and Half-Spaces. phys. stat. sol. (b) 30, 261-274. https://doi.org/10.1002/pssb.19680300132

Gemperlová, J., Paidar, V., Kroupa, F., 1989. Compatibility stresses in deformed bicrystals. Czechoslovak Journal of Physics 39, 427-446. https://doi.org/10.1007/BF01597801

Gemperlová, J., Saxl, I., 1968. Dislocations in an inhomogeneous anisotropic medium. Czech J Phys 18, 1085-1093. https://doi.org/10.1007/BF01690013

Han, X., Ghoniem, N.M., 2005. Stress field and interaction forces of dislocations in anisotropic multilayer thin films. Philosophical Magazine 85, 1205-1225. https://doi.org/10.1080/14786430412331331907

Hirth, J.P., Barkett, D.M., Lothe, J., 1979. Stress fields of dislocation arrays at interfaces in bicrystals. Philosophical Magazine A 40, 39-47. https://doi.org/10.1080/01418617908234831

Hirth, J.P., Lothe, J., 1982. Theory of dislocations. Wiley, New York.

Hwu, C., 2010. Anisotropic Elastic Plates. Springer US.

Juan, P.-A., Dingreville, R., 2017a. Mechanics of finite cracks in dissimilar anisotropic elastic media considering interfacial elasticity. Journal of the Mechanics and Physics of Solids 99, 1-18. https://doi.org/10.1016/j.jmps.2016.10.009

Juan, P.-A., Dingreville, R., 2017b. Elastic Green's Function in Anisotropic Bimaterials Considering Interfacial Elasticity. Journal of Elasticity 131, 277-296. doi: 10.1007/s 10659-017-9655-0

Kelly, A., Groves, G.W., 1970. Crystallography and crystal defects. Reading, Mass.: Addison-Wesley 
Kuan, H., Hirth, J.P., 1976. Dislocation pileups near the interface of a bimaterial couple. Materials Science and Engineering 22, 113-131.

Kuang, J.G., Mura, T., 1968. Dislocation Pile-up in Two-Phase Materials. Journal of Applied Physics 39, 109-119. https://doi.org/10.1063/1.1655715

Lee, T.C., Robertson, I.M., Birnbaum, H.K., 1990. An in situ Transmission Electron Microscope deformation study of the slip transfer mechanisms in metals. Metallurgical Transactions 21A, 2437-2447.

Leibfried, G., 1951. Verteilung von Versetzungen im statischen Gleichgewicht. Z. Physik 130, 214-226. https://doi.org/10.1007/BF01337695

Leknitskii, S.G., 1963. Theory of Elasticity of an Anisotropic Elastic Body. Holden-Day.

Li, J.C.M., Liu, G.C.T., 1967. Circular dislocation pile-ups. I. Strength of ultra-fine polycrystalline aggregates. Philosophical Magazine 15, 1059-1063.

Lubarda, V.A., 2017. An analysis of edge dislocation pileups against a circular inhomogeneity or a bimetallic interface. International Journal of Solids and Structures 129, 146-155. https://doi.org/10.1016/j.ijsolstr.2017.09.004

Mackenzie, J.K., Thomson, M.J., 1957. Some Statistics Associated with the Random Disorientation of Cubes. Biometrika 44, 205-210. https://doi.org/10.2307/2333253

Maugin, G.A., 2011. Configurational forces: thermomechanics, physics, mathematics and numerics. CRC Press, Modern Mechanics and Mathematics, Taylor and Francis, 2011.

Mitchell, T.E., Hecker, S.S., Smialek, R.L., 1965. Dislocation Pile-ups in Anisotropic Crystals. phys. stat. sol. (b) 11, 585-594. https://doi.org/10.1002/pssb.19650110210

Nakahara, S., Willis, J.R., 1973. Some remarks on interfacial dislocations. J. Phys. F: Met. Phys. 3, L249. https://doi.org/10.1088/0305-4608/3/12/001

Öveçoğlu, M.L., Doerner, M.F., Nix, W.D., 1987. Elastic interactions of screw dislocations in thin films on substrates. Acta Metallurgica 35, 2947-2957. https://doi.org/10.1016/0001-6160(87)90294-X

Pan, E., 2003. Three-Dimensional Green's Functions in Anisotropic Elastic Bimaterials With Imperfect Interfaces. Journal of Applied Mechanics 70, 180-190. doi:10.1115/1.1546243

Peach, M., Koehler, J.S., 1950. The Forces Exerted on Dislocations and the Stress Fields Produced by Them. Phys. Rev. 80, 436-439. https://doi.org/10.1103/PhysRev.80.436

Perrin, C., Berbenni, S., Vehoff, H., Berveiller, M., 2010. Role of discrete intragranular slip on lattice rotations in polycrystalline $\mathrm{Ni}$ : Experimental and micromechanical studies. Acta Materialia 58, 4639-4649.

Qu, J., Bassani, J.L., 1989. Cracks on bimaterial and bicrystal interfaces. Journal of the Mechanics and Physics of Solids 37, 417-433. https://doi.org/10.1016/0022-5096(89)90022-7

$\mathrm{Qu}$, J., Li, Q., 1991. Interfacial dislocation and its applications to interface cracks in anisotropic bimaterials. J Elasticity 26, 169-195. https://doi.org/10.1007/BF00041220

Richeton, T., Berbenni, S., 2013. Effects of heterogeneous elasticity coupled to plasticity on stresses and lattice rotations in bicrystals: A Field Dislocation Mechanics viewpoint. European Journal of Mechanics - A/Solids 37, 231-247. https://doi.org/10.1016/j.euromechsol.2012.06.010 
Schäfer, F., Weiter, L., Marx, M., Motz, C., 2016. Quantifying the grain boundary resistance against slip transfer by experimental combination of geometric and stress approach using stage-I-fatigue cracks. Philosophical Magazine 96, 3524-3551.

Shishvan, S.S., Mohammadi, S., Rahimian, M., Van der Giessen, E., 2011. Plane-strain discrete dislocation plasticity incorporating anisotropic elasticity. International Journal of Solids and Structures 48, 374-387.

Sigaeva, T., Schiavone, P., 2014. The effect of surface stress on an interface crack in linearly elastic materials. Mathematics and Mechanics of Solids 21, 649-656. https://doi.org/10.1177/1081286514534871

Stroh, A.N., 1962. Steady State Problems in Anisotropic Elasticity. Journal of Mathematics and Physics 41, 77-103. https://doi.org/10.1002/sapm196241177

Stroh, A.N., 1958. Dislocations and Cracks in Anisotropic Elasticity. Philosophical Magazine 3, 625-646. https://doi.org/10.1080/14786435808565804

Suo, Z., 1990. Singularities, interfaces and cracks in dissimilar anisotropic media. Proc. R. Soc. Lond. A 427, 331-358. https://doi.org/10.1098/rspa.1990.0016

Tiba, I., Richeton, T., Motz, C., Vehoff, H., Berbenni, S., 2015. Incompatibility stresses at grain boundaries in Ni bicrystalline micropillars analyzed by an anisotropic model and slip activity. Acta Materialia 83, 227-238.

Ting, T.C.T., 1992. Anatomy of Green's functions for line forces and dislocations in anisotropic media and in degenerate materials. Phys. Scr. 1992, 137. https://doi.org/10.1088/0031-8949/1992/T44/023

Ting, T.C.T., Barnett, D.M., 1993. Image force on line dislocations in anisotropic elastic half-spaces with a fixed boundary. International Journal of Solids and Structures 30, 313-323. https://doi.org/10.1016/0020-7683(93)90168-7

Ting, T.C.T., Chyanbin, H., 1988. Sextic formalism in anisotropic elasticity for almost non-semisimple matrix N. International Journal of Solids and Structures 24, 65-76. https://doi.org/10.1016/0020-7683(88)90099-6

Ting, T.T.C., 1996. Anisotropic Elasticity: Theory and Applications, Oxford Engineering Science Series. Oxford University Press, Oxford, New York.

Tucker, M.O., Crocker, A.G., 1968. The Plane Boundary in Anisotropic Elasticity, in: Mechanics of Generalized Continua, IUTAM Symposia. Springer, Berlin, Heidelberg, pp. 286-289. https://doi.org/10.1007/978-3-662-30257-6_34

Tucker, M.O., 1969. Plane Boundaries and Straight Dislocations in Elastically Anisotropic Materials. $\quad$ Philosophical Magazine 19, 1141-1159. https://doi.org/10.1080/14786436908228640

Vagera, I., 1971. Dislocation pile-up against a grain boundary in an anisotropic material. Czech J Phys 21, 77-82. https://doi.org/10.1007/BF01698087

Voskoboinikov, R.E., Chapman, S.J., Ockendon, J.R., Allwright, D.J., 2007. Continuum and discrete models of dislocation pile-ups. I. Pile-up at a lock. Journal of the Mechanics and Physics of Solids 55, 2007-2025.

Wagoner, R.H., 1981. Calculating Dislocation Spacings in Pile-Ups at Grain Boundaries. Metallurgical Transactions A 12, 2015-2023. https://doi.org/10.1007/BF02644170 
Wang, H.Y., Wu, M.S., Fan, H., 2007. Image decomposition method for the analysis of a mixed dislocation in a general multilayer. International Journal of Solids and Structures 44, 1563-1581. doi:10.1016/j.jijsolstr.2006.06.042 